\title{
The faint radio sky: VLBA observations of the COSMOS field ${ }^{\star}$
}

\author{
N. Herrera Ruiz ${ }^{1}$, E. Middelberg ${ }^{1}$, A. Deller ${ }^{2,3}$, R. P. Norris ${ }^{4,5}$, P. N. Best ${ }^{6}$, W. Brisken ${ }^{7}$, E. Schinnerer ${ }^{8}$, V. Smolčić ${ }^{9}$, \\ I. Delvecchio ${ }^{9}$, E. Momjian ${ }^{7}$, D. Bomans ${ }^{1}$, N. Z. Scoville ${ }^{10}$, and C. Carilli ${ }^{7}$
}

1 Astronomisches Institut, Ruhr-Universität Bochum, Universitätstrasse 150, 44801 Bochum, Germany e-mail: herrera@astro.rub.de

2 The Netherlands Institute for Radio Astronomy (ASTRON), 7991 Dwingeloo, The Netherlands

3 Centre for Astrophysics and Supercomputing, Swinburne University of Technology, PO Box 218, Hawthorn, VIC 3122, Australia

${ }^{4}$ CSIRO Australia Telescope National Facility, PO Box 76, Epping, NSW 1710, Australia

5 Western Sydney University, Locked Bag 1797, Penrith South, NSW 1797, Australia

${ }^{6}$ SUPA, Institute for Astronomy, Royal Observatory Edinburgh, Blackford Hill, Edinburgh EH9 3HJ, UK

7 National Radio Astronomy Observatory, PO Box O, Socorro, NM 87801, USA

8 MPI for Astronomy, Königstuhl 17, 69117 Heidelberg, Germany

9 Department of Physics, Faculty of Science, University of Zagreb, Bijenička cesta 32, 10000 Zagreb, Croatia

10 California Institute of Technology, MC 249-17, 1200 East California Boulevard, Pasadena, CA 91125, USA

Received 12 May 2017 / Accepted 13 July 2017

\begin{abstract}
Context. Quantifying the fraction of active galactic nuclei (AGN) in the faint radio population and understanding their relation with star-forming activity are fundamental to studies of galaxy evolution. Very long baseline interferometry (VLBI) observations are able to identify AGN above relatively low redshifts $(z>0.1)$ since they provide milli-arcsecond resolution.

Aims. We have created an AGN catalogue from 2865 known radio sources observed in the Cosmic Evolution Survey (COSMOS) field, which has exceptional multi-wavelength coverage. With this catalogue we intend to study the faint radio sky with statistically relevant numbers and to analyse the AGN - host galaxy co-evolution, making use of the large amount of ancillary data available in the field.

Methods. Wide-field VLBI observations were made of all known radio sources in the COSMOS field at $1.4 \mathrm{GHz}$ to measure the AGN fraction, in particular in the faint radio population. We describe in detail the observations, data calibration, source detection and flux density measurements, parts of which we have developed for this survey. The combination of number of sources, sensitivity, and area covered with this project are unprecedented.

Results. We have detected 468 radio sources, expected to be AGN, with the Very Long Baseline Array (VLBA). This is, to date, the largest sample assembled of VLBI detected sources in the sub-mJy regime. The input sample was taken from previous observations with the Very Large Array (VLA). We present the catalogue with additional optical, infrared and X-ray information.

Conclusions. We find a detection fraction of $20 \pm 1 \%$, considering only those sources from the input catalogue which were in principle detectable with the VLBA (2361). As a function of the VLA flux density, the detection fraction is higher for higher flux densities, since at high flux densities a source could be detected even if the VLBI core accounts for a small percentage of the total flux density. As a function of redshift, we see no evolution of the detection fraction over the redshift range $0.5<z<3$. In addition, we find that faint radio sources typically have a greater fraction of their radio luminosity in a compact core $-\sim 70 \%$ of the sub-mJy sources detected with the VLBA have more than half of their total radio luminosity in a VLBI-scale component, whereas this is true for only $\sim 30 \%$ of the sources that are brighter than $10 \mathrm{mJy}$. This suggests that fainter radio sources differ intrinsically from brighter ones. Across our entire sample, we find the predominant morphological classification of the host galaxies of the VLBA detected sources to be early type (57\%), although this varies with redshift and at $z>1.5$ we find that spiral galaxies become the most prevalent (48\%). The number of detections is high enough to study the faint radio population with statistically significant numbers. We demonstrate that wide-field VLBI observations, together with new calibration methods such as multi-source self-calibration and mosaicing, result in information which is difficult or impossible to obtain otherwise.
\end{abstract}

Key words. catalogs - galaxies: active - radio continuum: galaxies

\section{Introduction}

The main motivation of studying faint radio sources is to understand how active galactic nuclei (AGN) and star formation evolve through cosmic time. In particular, AGN appear to be fundamental players in galaxy evolution and star formation, which makes it necessary to determine where an AGN is present. Radio surveys are indispensable components of large multiwavelength

\footnotetext{
* The full Tables 2 and 3 are only available at the CDS via anonymous ftp to cdsarc.u-strasbg. fr (130.79.128.5) or via http://cdsarc.u-strasbg.fr/viz-bin/qcat?J/A+A/607/A132
}

studies since they are not affected by dust and they can detect the non-thermal radiation from AGN.

A strongly debated topic in astrophysics related to AGN and star formation interplay is the suggested link between accretion activity in AGN and star-forming activity of the host galaxy by AGN "feedback" (e.g. Best et al. 2006; Croton et al. 2006). In recent studies, two different accretion modes have been put forward for AGN-host galaxy co-evolution (e.g. Best et al. 2014): the cold-mode (radiatively efficient) and the hot-mode (radiatively inefficient). The cold-mode AGN are typically associated with star-forming galaxies and low mass black holes fuelled by 
cold gas of a thin, optically thick accretion disk with a high accretion rate (e.g. Heckman \& Best 2014; Norris et al. 2012; Smolčić et al. 2009). The hot-mode AGN are primarily associated with massive black holes hosted by elliptical galaxies and likely fuelled by hot gas of the halo leading to a slow growth of the black hole with a low accretion rate and a low or nonexistent star formation (e.g. Bower et al. 2006; Schawinski et al. 2009; Dubois et al. 2013). The main result of this energetic process is the outflow of collimated jets. For a more detailed description of these two modes, see Heckman \& Best (2014). Although this division of the two modes seems to work fine at low redshifts, it might not be right at high redshifts. Rees et al. (2016) investigated the host galaxy properties of a sample of radio-loud AGN and found that the majority of $z>1.5$ radio-AGN are hosted by star-forming galaxies. Zinn et al. (2013) suggested that both cold- and hot-mode mechanisms are important and showed that the star formation rate is correlated with radio jet power. Nevertheless, the specific astrophysics behind this relation are still not well understood.

Strazzullo et al. (2010) analysed the rest-frame $U-B$ versus $B$ colour-magnitude diagram of their radio selected sample and found that most of their sources were located in an intermediate location and not in two different locations, suggesting that at faint flux densities a simple classification between AGN or star-forming galaxies might not be appropriate. The properties of this intermediate location are not well understood yet, and it is composed of a mixed population of AGN, star-forming and composite galaxies where star formation and activity from the nucleus both play an important role. In addition, it has been shown in several cases that galaxies with a spectral energy distribution (SED) typical of a star-forming galaxy actually have the radio luminosity or morphology of an AGN. Norris (2009) suggest that such sources represent a class of AGN buried deeply inside a dusty star-forming galaxy, appearing to be an increasingly common phenomenon at high redshifts $(z \gtrsim 1)$.

It is therefore of considerable interest to produce AGN catalogues. However, it is usually difficult or impossible to distinguish between AGN and star-forming galaxies because radio observations are typically carried out with interferometers such as the Jansky Very Large Array or the Australia Telescope Compact Array. Because of their limited baseline lengths of several $\mathrm{km}$ or several tens of $\mathrm{km}$, these instruments are equally sensitive to radio emission from either process. Fortunately, a relatively direct way to identify which galaxies do have radioactive AGN is a detection with very long baseline interferometry (VLBI) observations. VLBI baselines are typically a few thousand $\mathrm{km}$ long, resulting in an angular resolution of the order of milli-arcsec. The brightness temperature a body needs to have to be detected using VLBI is around $10^{6} \mathrm{~K}$, which generally can only be reached by the non-thermal emission processes in AGN (Condon 1992). At a redshift of 0.1, the actual diameter of an object that can be resolved with the Very Long Baseline Array (VLBA) is 10 pc. Kewley et al. (2000) show that in compact objects such brightness temperatures can only be achieved by AGN activity which makes this technique a powerful method to cleanly separate AGN from star-forming galaxies.

The main disadvantage of VLBI is its tiny field of view, which covers a radius of only around $5 \mathrm{arcsec}$ at $\mathrm{GHz}$ frequencies. Consequently, this technique has historically been incompatible with large field observations, targeting a significant number of sources. However, a new technique has been developed to deal with this problem, the so-called "wide-field VLBI" technique (Garrett et al. 1999), whose main objective is to make the field of view in VLBI observations as wide as possible.
Garrett et al. (2001) presented deep, wide-field VLBI observations at $1.6 \mathrm{GHz}$ of the Hubble deep field (HDF) region. They detected two radio sources at the $5 \sigma$ level and a third radio source was detected at the $4 \sigma$ level. Garrett et al. (2005) conducted deep, wide-field VLBI observations at $1.4 \mathrm{GHz}$ of an area of the sky located within the NOAO Bootes field. They observed 61 sources and detected nine sources above the $6 \sigma$ level. Lenc et al. (2008) performed the first wide-field VLBI survey at $90 \mathrm{~cm}$. They targeted 618 sources in an area consisting of two overlapping fields centred on the quasar J0226+3421 and the gravitational lens $\mathrm{B} 0218+357$ and detected 27 sources out of the 272 detectable sources. Chi et al. (2013) carried out wide-field VLBI observations of the Hubble deep field north (HDF-N) and flanking fields (HFF). They observed 92 known radio sources with a global VLBI array at $1.4 \mathrm{GHz}$ and detected 12 sources above the $5 \sigma$ level. The development of wide-field VLBI was limited by the spectral and temporal resolution of the early generation of hardware correlators. To make this technique feasible, important progress in computer technology was required and the introduction of software correlators played a decisive role (Deller et al. 2007, 2011).

With the improvement in sensitivity of radio interferometers, the minimum of brightness temperature needed for a source to be detected can occasionally be reached by star-forming activity, radio supernovae or gamma-ray bursts. However, the luminosity of star formation quickly drops below the detection threshold when the galaxies are located beyond a redshift of 0.1 , where almost all of our targets are located. Furthermore, the transient events are exceedingly rare: after $\sim 30 \mathrm{yr}$ of observations only $\sim 50$ supernovae have been detected at radio wavelengths, none of which are type Ia (the most powerful ones; Lien et al. 2011), and from a sample of 304 gamma-ray burst observed with radio telescopes (during $14 \mathrm{yr}$ ) the fractional detection rate of radio afterglows is about $30 \%$ (Chandra \& Frail 2012). Therefore, we are confident that our sample of VLBI detected radio sources constitutes a pure sample of radio-active AGN.

We have observed 2865 known radio sources from Schinnerer et al. (2010) with the VLBA in the Cosmic Evolution Survey (COSMOS) field. COSMOS is an astronomical survey designed to probe the formation and evolution of galaxies as a function of cosmic time and large-scale structural environment (Scoville et al. 2007). The COSMOS field is located at $\operatorname{RA}(\mathrm{J} 2000)=10: 00: 28.6$ and $\operatorname{Dec}(\mathrm{J} 2000)=+02: 12: 21.0$ and is suitable to study the radio AGN-host galaxy interplay, since it is certainly the most comprehensive extragalactic survey to date. COSMOS includes very sensitive radio, sub-mm, infrared, optical and X-ray data from diverse facilities ${ }^{1}$ and provides a unique multi-wavelength coverage over an area as large as $2 \mathrm{deg}^{2}$. Moreover, it is ideally suited to study the faint radio sky, since the COSMOS field is mostly lacking even moderately strong radio sources.

In this paper, we describe the VLBA observations and we present the resulting AGN catalogue. We made use of several specialised wide-field VLBI techniques such as mosaicing, multi-source self-calibration, and primary beam corrections, to generate milli-arcsecond scale resolution images with a sensitivity of tens of $\mu \mathrm{Jy}$. We have previously used widefield VLBI observations to carry out similar observations for a wide range of flux densities (Middelberg et al. 2011, 2013; Deller \& Middelberg 2014), demonstrating the feasibility of the process.

http://cosmos.astro.caltech.edu/page/datasets 
The structure of this paper is as follows. In Sect. 2 we describe the details of the observations and the procedure of the data calibration. We present the catalogue of the VLBA detected sources in Sect. 3. In Sect. 4 the results of the observations are reported and discussed. In Sect. 5 we summarise the conclusions derived by the present project.

Throughout this paper, we assume a flat $\Lambda \mathrm{CDM}$ cosmology with $H_{0}=67.3 \mathrm{~km} \mathrm{~s}^{-1} \mathrm{Mpc}^{-1}, \Omega_{\mathrm{M}}=0.31$ and $\Omega_{\Lambda}=$ 0.69 (according to the recent Planck results published by Planck Collaboration XVI 2014).

\section{Observations and data calibration}

In this section, we describe our sample, the characteristics of the observations and the procedures followed to calibrate the data. We also present the obtained sensitivity map and the description of the criteria adopted for source extraction. For clarity, we establish the nomenclature used here:

- the term "target" refers to an object to be observed with the VLBA;

- the term "source" refers to a physical source;

- the term "component" refers to a connected region of radio emission;

- the term "pointing" refers to one of the 23 regions, within which objects were targeted during an observing run;

- the term "epoch" refers to six hours observation of one pointing;

- the term "phase centre" refers to a location within a telescope's beam where correlation can focus;

- the term "calibration file" refers to the data set of each epoch, containing calibration information from the phase-referencing source $\mathrm{J} 1011+0106$ and the fringe-finder 4C 39.25.

\subsection{Sample}

We took the target positions for our sample from the VLA catalogue of Schinnerer et al. (2010). Because the individual fields of view in VLBI observations are smaller than the extension of the source as seen on arcsec scales, we decided to target each component of the sources classified as multi-component by the initial catalogue. In particular, from the 2865 radio sources, 131 were multi-component sources, yielding a total of 3293 targets.

\subsection{Observations}

We observed 3293 targets in the COSMOS extragalactic field with the VLBA at a central frequency of $1.54 \mathrm{GHz}$ over 23 pointings between February 2012 and January 2013. To compensate for the reduced sensitivity at the edge of the pointings, we used a pattern of overlapping pointings (radius $\sim 15^{\prime}$ ), which is standard practice with compact interferometers (see Fig. 1). For this reason, most of the targets were observed several times. Each pointing was observed twice for $6 \mathrm{~h}$, to increase observing time at high elevations and to allow for greater scheduling flexibility. This resulted in 46 individual observing epochs. During each epoch, the target field was repeatedly observed for $4.5 \mathrm{~min}$, followed by a 1 min observation of the phase-referencing source J1011+0106. For data consistency checks, the fringe-finder 4C 39.25 was observed every $2 \mathrm{~h}$. In summary, the on-source integration time per pointing was roughly $8.5 \mathrm{~h}$, with the maximum number of overlapping pointings being seven. Eight 32-MHz bands were observed in two circular polarisations, requiring a recording rate of

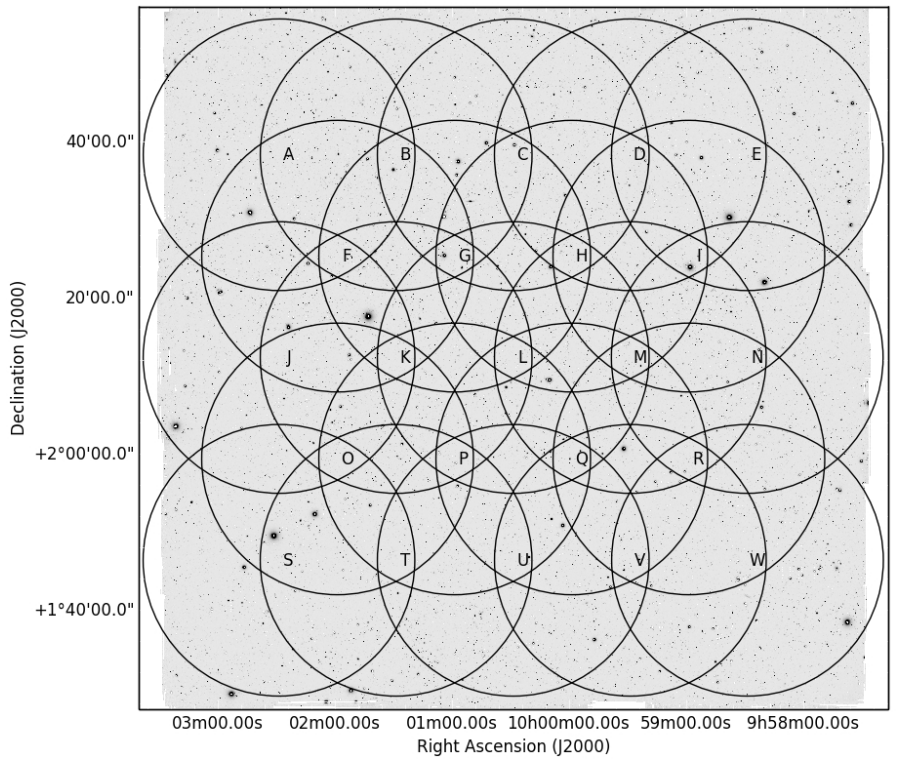

Fig. 1. The 23 pointings representing the design of our VLBA observations of the COSMOS field. The black circles denote the radius within which sources were targeted during an observing run $\left(\sim 15^{\prime}\right)$. The letters denote the identification for each pointing. The background greyscale image is a mosaic of COSMOS Subaru $i$-band data ${ }^{2}$.

2 Gbps. A minimum number of nine VLBA antennas was scheduled to achieve the required sensitivity.

Historically, VLBI observations have provided the highest resolution in astronomy with the drawback of covering only tiny fields of view (around 5 arcsec radius at $\mathrm{GHz}$ frequencies) as a result of the high fringe rates implied in VLBI observations. This has made them unsuitable for observing large fields with a considerable number of objects. However, a new multi-phase centre mode has been developed for the VLBA DiFX correlator in operation at the VLBA (Deller et al. 2007). In this mode, the initial correlation is carried out with high time and frequency resolution. The visibilities are subsequently phase-shifted to the other phase centres, before they are averaged in time and frequency and written to disk. This minimises the effects of time and bandwidth averaging, while at the same time the resulting data sets are kept comparatively small. Thus, within a region confined by the individual antenna's primary beams one can position numerous phase centres. This mode can be used to image hundreds of objects in a single observing run (Deller et al. 2011). In our case, an average of 450 VLA sources per pointing were targeted. The raw dataset size of each individual target was $350 \mathrm{MB}$, yielding around $158 \mathrm{~GB}$ per epoch on average. Since we had 46 epochs, the total amount of raw data from our observations was $\sim 7 \mathrm{~TB}$, indicating that processing was a significant computing effort.

\subsection{Data calibration}

We have calibrated the data using the Astronomical Image Processing System (AIPS, Greisen 2003; Fomalont 1981) following standard procedures used in phase-referenced VLBI observations together with specialised techniques developed for wide-field VLBI observations. Our script to calibrate the data has been written in ParselTongue (AIPS Talking Python, Kettenis et al. 2006). The details of our calibration procedure are

2 http://irsa.ipac.caltech.edu/data/Cosmos/ 
described as follows. The specialised steps of wide-field VLBI are referred to as "non-standard calibration steps".

\subsubsection{Loading and initial preparation of the uv data}

We loaded the data into AIPS using the task FITLD. If there were redundancies in prior calibration information, such as system temperature and gain curves, on the same source and/or antenna in a given data set, we used the procedure MERGECAL to remove the redundant information. We sorted the visibility data set into time-baseline order using the task UVSRT.

This is a non-standard calibration step. Each epoch consists of one calibration file and data sets containing measurements of each target. First, we use the calibration file to find the corrections to calibrate the data.

\subsubsection{First corrections}

Since the parallactic angle between the calibrator and target is different at different stations, we corrected for this phase term using the AIPS task CLCOR.

The Earth orientation parameters (EOPs) used at correlation time are later updated and refined, resulting in a change of observed visibility phase. To correct for this, we obtained updated EOPs from the USNO server ${ }^{3}$ and applied corrections using the task CLCOR.

The ionosphere can cause unmodelled dispersive delays that we corrected using measurements ${ }^{4}$ of total electron content (TEC) and the task TECOR.

We corrected the amplitude offsets arising from sampler errors at the stations, typically of order 5-10\%, using the task ACCOR.

\subsubsection{Phase, bandpass and amplitude calibration}

Residual delays, rates and phases were measured using data from the phase calibrator J1011+0106 and the fringe-finder 4C 39.25, using the task FRING. We used a solution interval of two minutes and averaged the data in each IF of $32 \mathrm{MHz}$.

A recent VLBA Scientific Memo ${ }^{5}$ reports that the then standard amplitude calibration of the VLBA caused amplitude errors of order $25-30 \%$. The memo recommends to form a model bandpass using the full band and power normalisation and to scale the data by a small factor to make the calibrated autocorrelation values unity. We implemented this procedure using the task BPASS on the fringe-finder, and the new AIPS task ACSCL to deal with the small offset from unity amplitude in the calibrated autocorrelations.

We carried out amplitude calibration using the antennas' system temperature $\left(T_{\text {sys }}\right)$ measurements and known gain curves with the task APCAL. $T_{\text {sys }}$ depends on the antenna zenith angles, $\Theta_{z}$, and follows approximately $1 / \cos \left(\Theta_{z}\right)$. Because of the low declination of the field $\left(2^{\circ}\right)$ and the limited window of $6 \mathrm{~h}$ observing time, the zenith angle (and therefore antenna elevation) at most stations was roughly constant throughout the observations and the $T_{\text {sys }}$ value were expected to vary only little. Typical $T_{\text {sys }}$ were found to be of order $30 \mathrm{~K}$, and values exceeding $50 \mathrm{~K}$ were deemed to indicate errors. These values were flagged,

\footnotetext{
3 http://gemini.gsfc.nasa.gov/solve_save/usno_finals. erp

$4 \mathrm{ftp}: / /$ cddis.gsfc.nasa.gov/gps/products/ionex

5 http://library.nrao.edu/public/memos/vlba/sci/VLBAS_ 37.pdf
}

which resulted in the respective visibility data not being used in subsequent processing steps. We consider that the surface brightness (SB) error resulting from calibration is of the order of $10 \%$ (Middelberg et al. 2013). We used this error later to calculate the errors of the VLBA flux densities (see Sect. 2.6).

\subsubsection{Flagging}

We edited the data using a flagging programme written by us and implementing the procedures described in Middelberg (2006), which compare the median amplitude in each channel to the median of an RFI-free reference channel. Around 4-5\% of the data were flagged.

\subsubsection{Multi-source self-calibration}

This is a non-standard calibration step. In phase-referenced observations, images have reduced coherence due to ionospheric and atmospheric turbulence. We have followed a two-stage selfcalibration procedure to correct for residual phase and amplitude errors. This procedure is described in Middelberg et al. (2013). In general terms, the first stage consists of amplitude and phase self-calibration of the data using a model of the phase calibrator and the second step consists of using detected targets with signal-to-noise ratio $(\mathrm{S} / \mathrm{N})$ larger than 7 to apply multi-source self-calibration to phase self-calibrate the data. The idea of the multi-source self-calibration is that while individual targets are not sufficiently strong to be used in self-calibration, a combination of the strongest few targets in each epoch in general is. The structure and position of these targets can be divided out using the CLEAN models obtained in imaging. The improvements achieved with this procedure were notable and one example is illustrated in Fig. 2, where we can see that the peak flux density has increased and the sidelobe level is reduced. After this procedure, we found between five and ten more detected sources per epoch, in comparison to the number of detected sources found before it (a median of $\sim 15$ per epoch). Radcliffe et al. (2016) tested a calibration algorithm based on multi-source selfcalibration on a $1.6 \mathrm{GHz}$ wide-field VLBI data set of the HDF-N and the HFF and found great improvements in dynamic range for all the detected sources.

\subsubsection{Primary beam correction}

This is a non-standard calibration step. We copied the amplitude and phase corrections from the calibration file to the rest of the data sets considering that the phase response of a VLBA antenna is constant across the primary beam. However, the apparent flux density of a source can be attenuated by up to $50 \%$ due to the amplitude response through the primary beam. Therefore, we corrected all data sets for primary beam attenuation. This correction is described in detail by Middelberg et al. (2013), who carried out observations using a pattern of pointing positions around $3 \mathrm{C} 84$ with the VLBA at $1.4 \mathrm{GHz}$ to measure the primary beam response of the antennas. Moreover, we also corrected the offset between the beam patterns of the two polarizations (VLBA beam squint). We carried out this step using the AIPS task CLVLB.

\subsubsection{Data combination}

This is a non-standard calibration step. Targets in the overlap region of pointings have been observed several times. Once we 
N. Herrera Ruiz et al.: The faint radio sky: VLBA observations of the COSMOS field
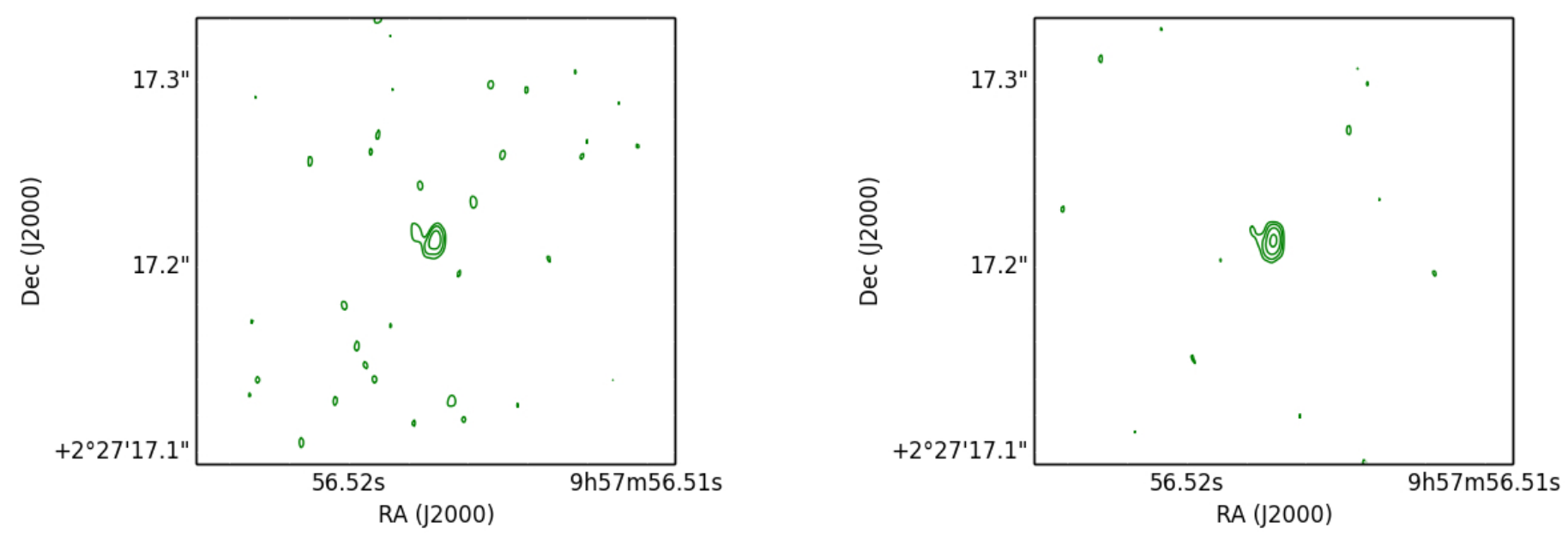

Fig. 2. Contour plots of a target before applying multi-source self-calibration (left) and after applying multi-source self-calibration (right). The peak flux density of the left panel image is $1.2 \mathrm{mJy}$ and the rms is $58 \mu \mathrm{Jy}$. The peak flux density of the right panel image is $1.6 \mathrm{mJy}$ and the rms is $55 \mu \mathrm{Jy}$. Positive contours start at three times the rms level of the image and increase by a factor of two. The image is uniformly-weighted. The peak flux density has increased and the sidelobe level is reduced.

considered the calibration of all individual data sets complete, the last step was to combine the data of each target observed in separate epochs to reach maximum sensitivity. We used the task DBCON to combine the calibrated data. External conditions such as weather, atmosphere or ionosphere limited the accuracy of the target positions. As a result, we observed slight variations in the position of the target between epochs. To handle this, we computed the median of the variation for each epoch and pointing. To compute the median offset for each epoch we compared the two epochs of the same pointing, taking one of them as reference. To compute the median offset for each pointing we compared each pointing to the reference pointing, which we chose to be the one in the middle of the design (pointing $\mathrm{L}$, Fig. 1). In every epoch, there were about 450 targets, of which a median of 20 presented a peak flux density exceeding seven times the rms noise. We recorded the position of the peak flux density (obtained by fitting a quadratic function to a $3 \times 3$ map array) of these 20 targets. To compute the median offset for each pointing, we analysed first the pointings overlapping with the reference pointing (pointing $\mathrm{L}$ ). For each of these pointings, we computed the difference of the position of each target which was also observed in the pointing L. Then, we examined the pointings not overlapping the reference pointing $\mathrm{L}$. We calculated the variations considering the ones of the pointing closer to the reference pointing, and subsequently added to it. To illustrate this, we will give an example for the pointings L-M-N, where we calculated the median offset in RA and Dec of pointing $M$ relative to $L$, and then we added the median offset of pointing $\mathrm{N}$ relative to $\mathrm{M}$. We made eight detections common to the pointings $\mathrm{L}$ and $\mathrm{M}$ and recorded the positions of each of these detections. The median offset for the pointing $\mathrm{M}$ in right ascension $\left(\triangle \mathrm{RA} \mathrm{A}_{\mathrm{M}}\right)$ and in declination $\left(\Delta \mathrm{Dec}_{\mathrm{M}}\right)$ was 0.6 mas and 2.7 mas, respectively. Since there were no overlapping regions between the pointing $\mathrm{L}$ and the pointing $\mathrm{N}$, we used the positions of the pointing $\mathrm{M}$ to calculate the variations of the pointing $\mathrm{N}$. In this case, we observed ten detections in both pointings $\mathrm{M}$ and $\mathrm{N}$. We added the median obtained from the variation in right ascension and declination to the variations obtained from pointings L-M, resulting in $\Delta \mathrm{RA}_{\mathrm{N}}=0.6$ mas and $\Delta \mathrm{Dec}_{\mathrm{N}}=$ 1.9 mas. In summary, we used the following equations for this example:

$$
\begin{aligned}
\Delta \mathrm{RA}_{\mathrm{L}} & =0 ; \Delta \mathrm{Dec}_{\mathrm{L}}=0, \\
\Delta \mathrm{RA}_{\mathrm{M}} & =\mathrm{RA}_{\mathrm{L}}-\mathrm{RA}_{\mathrm{M}} ; \Delta \mathrm{Dec}_{\mathrm{M}}=\mathrm{Dec}_{\mathrm{L}}-\mathrm{Dec}_{\mathrm{M}}, \\
\Delta \mathrm{RA}_{\mathrm{N}} & =\Delta \mathrm{RA}_{\mathrm{M}}+\left(\mathrm{RA}_{\mathrm{M}}-\mathrm{RA}_{N}\right) ; \Delta \operatorname{Dec}_{\mathrm{N}} \\
& =\Delta \operatorname{Dec}_{\mathrm{M}}+\left(\operatorname{Dec}_{\mathrm{M}}-\operatorname{Dec}_{\mathrm{N}}\right),
\end{aligned}
$$

where $\mathrm{RA}_{\text {(pointing_ID) }}$ and $\operatorname{Dec}_{\text {(pointing_ID) }}$ are the measured positions of the targets in the pointing. Once we calculated the variations, we corrected the position of all the targets using the task UVFIX. Table 1 contains the median offsets in right ascension and declination for each epoch and pointing. The relative astrometric accuracy of the VLBA is of the order of 10 micro-arcsec ${ }^{6}$. Nevertheless, we carried out the phase calibration with the nodding calibration to the phase reference source located $\sim 3^{\circ}$ away. Therefore, the absolute position uncertainties of the sources after phase calibration will be dominated by the residual astrometric error over a few degrees. For any given pointing, the seed positions for multi-source self-calibration is likely to be off by up to a few mas, which is exactly what we see. Deller et al. (2016) measured the absolute position uncertainty of the reference position for their sources after phase referencing over a couple of degrees and found it to be $\sim 2$ mas at $1.4 \mathrm{GHz}$ at a declination of $-8^{\circ}$. The median of the variations for all the pointings discussed in this project is 0.7 mas in right ascension and 1 mas in declination. After correcting the positions of all the targets, we further measured the peak flux density, resulting in a median of $0.02 \%$ increase of the peak flux density and three more detections (considering as detection those sources with $\mathrm{S} / \mathrm{N}$ larger than 5.5; for further details see Sect. 2.5.1).

\subsubsection{Imaging}

For source detection, we made naturally-weighted images to maximise sensitivity using the task IMAGR. Since the VLA observations had arcsecond resolution and our VLBA observations

\footnotetext{
6 https://science.lbo.us/facilities/vlba
} 
Table 1. Corrections of the target positions for each epoch and pointing

\begin{tabular}{c|rr|rr}
\hline \hline & \multicolumn{2}{c|}{ Offset epoch } & \multicolumn{2}{c}{ Offset pointing } \\
\hline Pointing & $\begin{array}{r}\text { RA } \\
(\mathrm{mas})\end{array}$ & $\begin{array}{r}\text { Dec } \\
(\mathrm{mas})\end{array}$ & $\begin{array}{r}\text { RA } \\
(\mathrm{mas})\end{array}$ & $\begin{array}{r}\text { Dec } \\
(\mathrm{mas})\end{array}$ \\
\hline $\mathrm{A}$ & -0.49 & 2.06 & -0.22 & -0.33 \\
$\mathrm{~B}$ & -0.04 & 0.68 & 0.83 & 1.29 \\
$\mathrm{C}$ & -0.65 & 0.85 & 1.78 & 2.62 \\
$\mathrm{D}$ & -1.69 & -1.03 & 1.76 & 0.50 \\
$\mathrm{E}$ & -1.42 & 2.60 & 1.40 & 0.52 \\
$\mathrm{~F}$ & -0.05 & -1.49 & -0.40 & 2.85 \\
$\mathrm{G}$ & 0.43 & -3.09 & -0.04 & 1.65 \\
$\mathrm{H}$ & 0.51 & 1.60 & -0.30 & 0.26 \\
$\mathrm{I}$ & -1.42 & -2.23 & 0.76 & 1.91 \\
$\mathrm{~J}$ & -1.14 & 2.66 & -0.95 & -0.16 \\
$\mathrm{~K}$ & -5.97 & -1.16 & 0.51 & 1.01 \\
$\mathrm{~L}$ & 2.08 & 1.19 & 0.00 & 0.00 \\
$\mathrm{M}$ & -0.06 & -1.94 & 0.60 & 2.69 \\
$\mathrm{~N}$ & -1.94 & -1.10 & 0.61 & 1.91 \\
$\mathrm{O}$ & -2.04 & -1.90 & -0.76 & 1.35 \\
$\mathrm{P}$ & 0.99 & 2.16 & -0.88 & -1.11 \\
$\mathrm{Q}$ & 1.98 & -1.48 & 0.71 & 0.52 \\
$\mathrm{R}$ & 0.12 & -0.93 & 0.53 & 1.04 \\
$\mathrm{~S}$ & -1.65 & -2.92 & 0.55 & 2.14 \\
$\mathrm{~T}$ & -2.14 & -2.20 & -1.23 & -0.61 \\
$\mathrm{U}$ & 3.35 & -2.33 & 1.03 & 0.01 \\
$\mathrm{~V}$ & 0.25 & -2.18 & 0.82 & 0.17 \\
$\mathrm{~W}$ & -2.59 & 4.62 & 0.39 & 2.40 \\
\hline
\end{tabular}

Notes. The corrections of each epoch refer to the offset between the two epochs of the same pointing. The corrections of each pointing refer to the offset between the pointing and the reference pointing $\mathrm{L}$.

have milli-arcsecond resolution, we expected the position of the targets to be offset from the VLA position. Therefore, we produced big images of $4096 \times 4096$ pixels with a pixel size of 1 mas. We cleaned the data until the first negative clean component was reached using a clean box rejecting a band of 100 pixels around the image edges due to commonly present spurious high values. The median of the restoring beam was $16.2 \times 7.3 \mathrm{mas}^{2}$.

For source flux density and position measurements we generated uniformly-weighted images. The uniform weighting gives better angular resolution at the expense of sensitivity. Moreover, the distribution of the VLBA antennas produces a plateau in the synthesised beam when natural weighting is used, and this plateau was previously found to significantly increase the recovered flux density, in particular at low S/N (e.g., Middelberg et al. 2013). We used the task IMAGR as explained in the previous step, changing the weighting option to uniform. The median of the restoring beam in this case was $12.4 \times 5.3 \mathrm{mas}^{2}$.

\subsection{Sensitivity map}

For an overview of the final sensitivity of our observations we computed the rms of all naturally-weighted images. These values were gridded into an image using linear interpolation between measurements to cover the region of the COSMOS VLA observations $\left(2 \mathrm{deg}^{2}\right)$. Figure 3 shows the sensitivity map obtained in this project, and the one obtained by Schinnerer et al. (2010) for comparison, since we designed our VLBA observations in order to achieve a similar rms distribution to the VLA observations.

\subsection{Source extraction}

Since the maximum sensitivity is reached in the naturally weighted images, we used these for source detection. We establish two main conditions to positively identify detected sources: i) the $\mathrm{S} / \mathrm{N}$ of the naturally-weighted image should be higher than 5.5 (see Sect. 2.5.1); ii) if the $\mathrm{S} / \mathrm{N}$ is lower than 7, the position of the VLBA detected source should be within 0 '"4 of the central part of the VLA radio contours or the optical counterpart (see Sect. 2.5.2). The steps followed to consider a detection as real are explained in detail in Sect. 2.5.3.

\subsection{1. $\mathrm{S} / \mathrm{N}$ threshold}

To minimise the number of false detections, we ran a test using only noise images to establish the $\mathrm{S} / \mathrm{N}$ threshold for the source detection. We make the noise images by imaging the sky $10^{\prime \prime}$ north of the target positions. Taking into account the low probability of finding a source $10^{\prime \prime}$ in declination away from our target, we can estimate the false-detection rate corresponding to several $\mathrm{S} / \mathrm{N}$ thresholds by measuring the peak flux density of these images. We found that the probability of finding a falsepositive together with the probability of having a chance detection within 0 .'.4 of the VLA position is $19 \%, 0.2 \%$ and $0.02 \%$ for a $\mathrm{S} / \mathrm{N}$ threshold of 5, 5.5 and 6 , respectively. The false-positive rate for a $\mathrm{S} / \mathrm{N}$ of 5 is too high (19\%), whereas for a S/N threshold of 6 is almost null $(0.02 \%)$ but with the drawback of missing some real detections. Therefore, we decided to consider 5.5 as the $\mathrm{S} / \mathrm{N}$ threshold, having a false-positive rate of only $0.2 \%$.

Considering a $\mathrm{S} / \mathrm{N}$ of 7 , we found a false detection rate of $0.03 \%$ in a $4096 \times 4096$ pixel image.

\subsubsection{Radio contours and optical counterparts}

We created cutout images of $15^{\prime \prime}$ from Hubble Space Telescope Advanced Camera for Surveys (HST-ACS), Subaru ( $r+$ band) and VLA from the NASA/IPAC Infrared Science Archive ${ }^{7}$ centred on each target position. We plot VLA contours of each target, starting at four times the rms noise level of the VLA image and increasing by a factor of two. Furthermore, we plot VLBA contours, starting at three times the rms noise level of the naturally-weighted image and increasing by a factor of $\sqrt{2}$. Figure 4 shows some examples of the optical counterparts and the radio contour plots of the targets.

Each pair of panels in Fig. 4 contains information about: i) optical counterpart (when the HST image was not available, the Subaru image ( $r$-band) was used); ii) VLA contours; iii) position of the VLBA peak flux density; iv) VLBA contours of the naturally-weighted image.

\subsubsection{Decision tree}

To decide if the detections were real, we passed each detection through a decision tree (see Fig. 5). The decision tree was created to minimise human interaction with the data and the probability

\footnotetext{
7 http://irsa.ipac.caltech.edu
} 

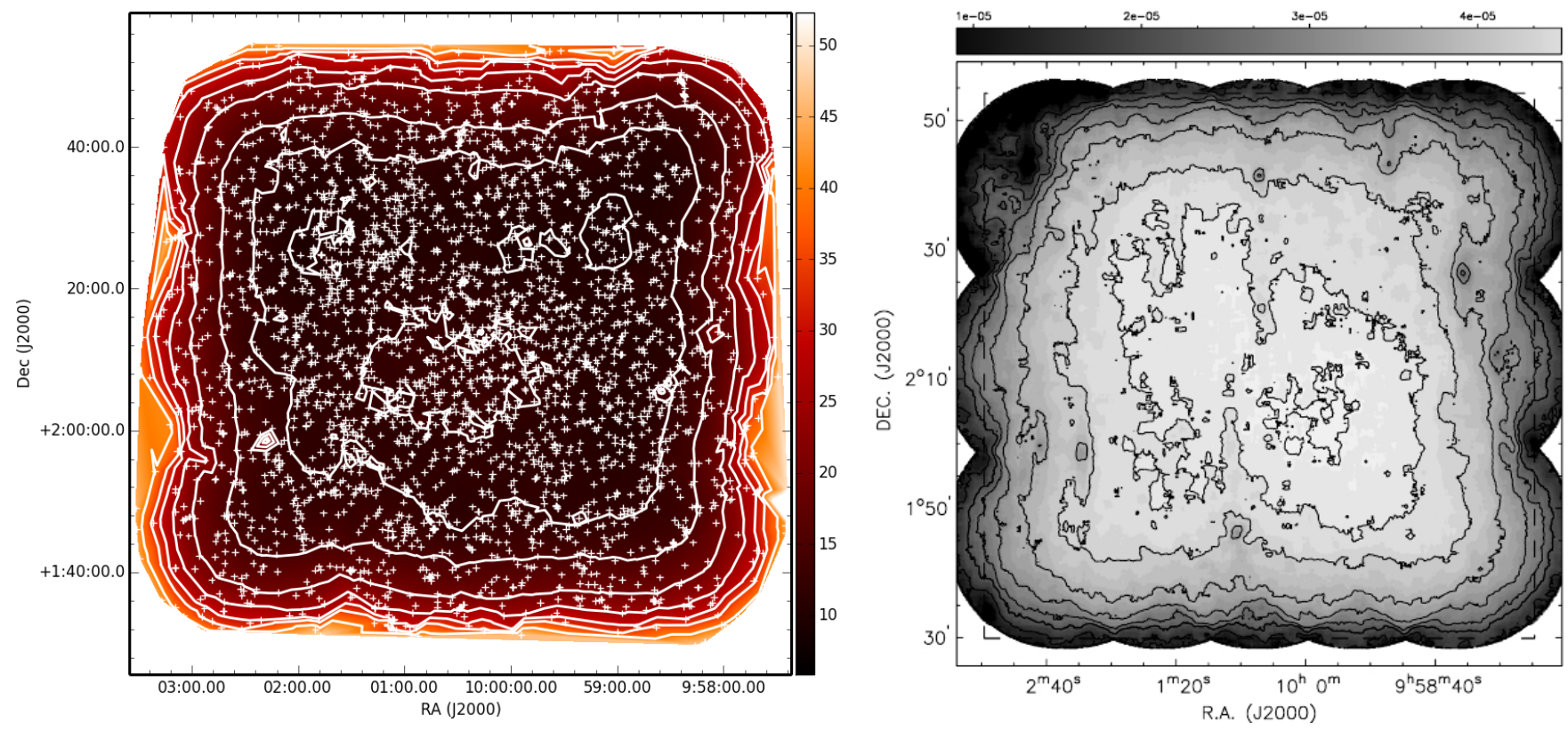

Fig. 3. Left panel: sensitivity map of the VLBA-COSMOS project. The colour bar represents the rms noise values in $\mu \mathrm{Jy} / \mathrm{beam}$. The white crosses show the target positions. White contours are drawn at 10, 12, 15, 20, 25, 30, 34, and $40 \mu \mathrm{Jy} /$ beam to match the contours in the VLA image. Right panel: Fig. 4 from Schinnerer et al. (2010), representing the sensitivity map of the VLA-COSMOS Deep project. The contours correspond to rms levels of $10,12,15,20,25,30,34$, and $40 \mu \mathrm{Jy} / \mathrm{beam}$. We can see that the rms distribution of the two images is in excellent agreement.

of having false detections. The steps of the decision tree are as follows:

1. The first step for a detected source to be passed through the decision tree was that it must present a peak flux density exceeding 5.5 times the local rms (see Sect. 2.5.1). A total of 710 sources satisfied this criterion.

2. If the $\mathrm{S} / \mathrm{N}$ was greater than 7 , we considered the detection as real, since this $\mathrm{S} / \mathrm{N}$ is high enough to neglect the false detection rate $(0.03 \%)$. A total of 366 sources satisfied this criterion.

3. If the $\mathrm{S} / \mathrm{N}$ was lower than 7 , we analysed the compactness of the detection. We considered a source compact when it was classified as unresolved and single-component source in the VLA catalogue of Schinnerer et al. (2010). By plotting the number of detections with $S / N>7$ versus the VLAVLBA position separation, we considered that a compact source must be located at a separation $<0$ '!4 (see Fig. 6), to consider it a real detection. A total of 83 sources satisfied this criterion.

4. If the source was considered not compact, then we checked it by eye (see Fig. 4). If the detection was coincident with the optical counterpart we considered it a real detection. If no optical counterpart was present, and the detection was located in the central part of the VLA contours, we considered the detection real. A total of 19 sources satisfied this criterion.

After passing our 3293 initial targets through the decision tree, we ended up with 468 detections. Considering the false detection rate when $S / N>7(0.03 \%)$ and when $5.5<S / N<7$ with the VLA-VLBA position separation being smaller than 0 "!4 $(0.2 \%)$, we estimate an overall number of false positives in the final catalogue of $<1$.

\subsection{VLBA flux density and position}

We ran $\mathrm{BLOBCAT}^{8}$ on the uniformly-weighted images to measure the flux density and position of the VLBA detected sources, since, as mentioned in Sect. 2.3, the angular resolution of the uniformly-weighted images is better and the plateau of the naturally-weighted images can overestimate the flux density of the source. BLOBCAT catalogues the flood filled islands of pixels (blobs) above a $\mathrm{S} / \mathrm{N}$ cutoff within a sea of noise, considering each island as a component of a single or multi-component source (Hales et al. 2012, 2014).

We define four parameters before running BLOBCAT: i) - -pasbe $=0.1$, since we consider the surface brightness (SB) error resulting from calibration to be $10 \%$; ii) -- ppe $=0.01$, we assume a SB pixellation error of $1 \%$ due to the well-sampled radio images; iii) -- cpeRA $=6.8 \mathrm{e}-4$; iv) -- cpeDec $=1.98 \mathrm{e}-3$. These two last parameters define the phase calibrator RA and Dec position errors ${ }^{9}$ (in $\operatorname{arcsec}$ ), respectively. Then, we run BLOBCAT to compute the position (RA, Dec), its uncertainty $(\triangle \mathrm{RA}, \Delta \mathrm{Dec})$, the rms, the peak flux density $\left(S_{\mathrm{p}, \mathrm{VLBA}}\right)$, its uncertainty $\left(\Delta S_{\mathrm{p}, \text { VLBA }}\right)$, the integrated flux density $\left(S_{\mathrm{i}, \text { VLBA }}\right)$ and its uncertainty $\left(\Delta S_{\mathrm{i}, \mathrm{VLBA}}\right)$ for each VLBA detected source. Each output was added to the catalogue derived from this work (Table 2), after being checked visually in order to remove the artefacts. We found a median of $\triangle \mathrm{RA}$ and $\Delta \mathrm{Dec}$ of 0.8 mas and 2.2 mas, respectively.

For multi-component sources a lower case letter was added to the ID of the source for each component. In these cases, a new line was added containing the original ID, the weighted average of the position, the rms of the uniformly-weighted image calculated with the AIPS verb imstat, the sum of the integrated flux density and its error (Table 2).

The weighted average of the position, $p$, was calculated as

$p=\sum\left(x_{n} \cdot f_{n}\right) / \sum f_{n}$

\footnotetext{
8 http://blobcat. sourceforge.net

9 https://www.lbo.us/vlba/astro/calib/
} 
C0090, VLA contours from 282.6 uJy, VLBA N_weight S/N 80.8 VLA max 14685.2, VLBA max 1850.9 (uJy). HST Image
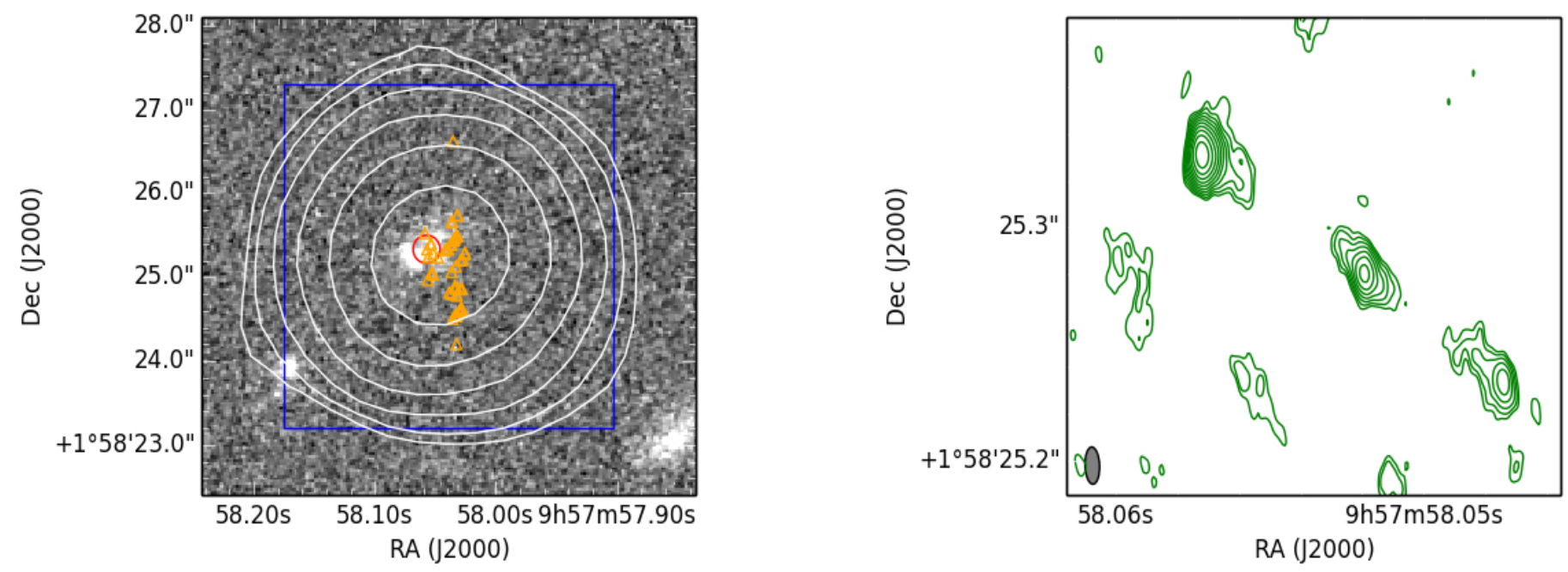

C1739, VLA contours from 50.0 uJy, VLBA N weight S/N 25.9 VLA max 398.9, VLBA max 313.7 (uJy). HST Image
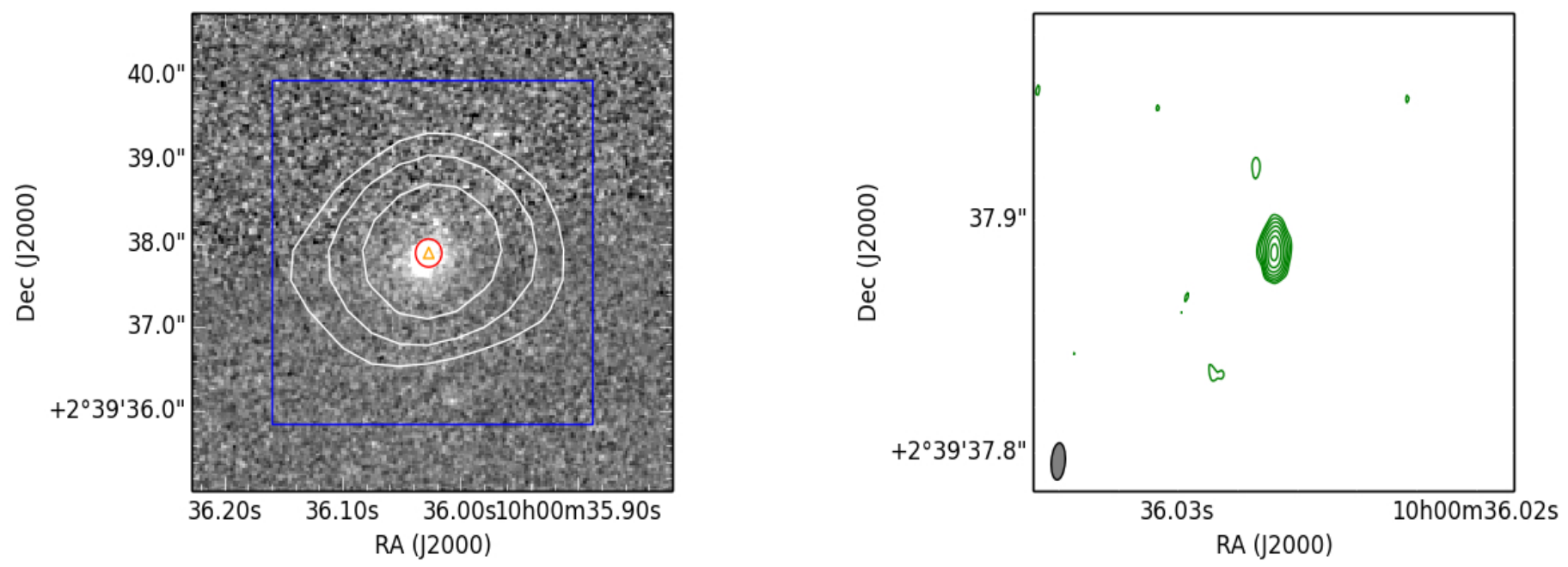

Fig. 4. Optical counterparts and contour plots of two VLBA detections. The header of each pair of panels contains: i) the source name used in the present project; ii) the rms noise value at which the VLA contours start; iii) the VLBA naturally-weighted image S/N; iv) the VLA peak flux density (in $\mu \mathrm{Jy}$ ); v) the VLBA peak flux density (in $\mu \mathrm{Jy}$ ); vi) the background greyscale image used (HST or Subaru). Left panel: background greyscale image is the HST/Subaru image of the VLBA detection counterpart. The blue square represents the 4 " $\times 4$ " VLBA image dimension. The white contours represent the VLA contours of the source, starting at four times the rms noise level of the VLA image and increasing by a factor of two. The red circle represents the VLBA peak flux density position. The orange triangles represent positions where the S/N of the VLBA naturally-weighted image is greater than 5.5. Right panel: green contours represent the VLBA detection contours, starting at three times the rms noise level of the naturally-weighted image and increasing by a factor of $\sqrt{2}$.

where $x$ is the position (RA or Dec), $f$ is the flux density and $n=$ $1,2, \ldots, i(i=$ number of components).

The error in the sum of the flux density, $\delta f$, corresponds to

$\delta f=\sqrt{\sum \delta f_{n}^{2}}$.

The high resolution of the VLBA data makes the position of the detected source more precise. Nevertheless, this position still has to match with the VLA position to some degree. Therefore, we compared the positions of the peak flux densities of the VLBA detected sources to the positions of their corresponding VLA targets and we calculated the separation between them. We found a median of the angular separation between the VLA target position and the VLBA detected source of 136 mas.
Figure 7 shows the relative positions between the VLA and the VLBA emission. Out of the 468 detections, 421 (90\%) are within a radius of 232 mas offset from the central position of the VLA target. Those detections with an offset greater than $1^{\prime \prime}$, have a S/N larger than 7, and so are clearly detected, but the VLA source was very extended.

\section{Catalogue}

We constructed a catalogue containing 468 sources expected to be AGN, of which 14 are considered as multi-component sources (see Table 2). The column entries are the following:

- Column (1) - Source name used in the present project (ID). 


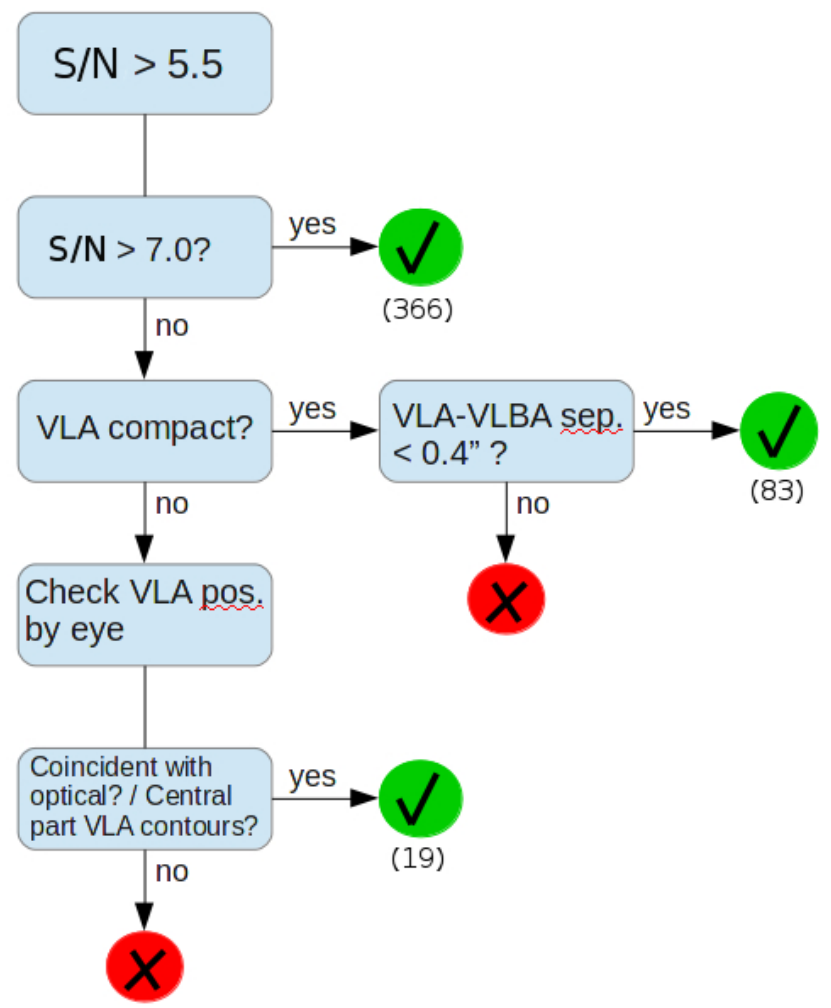

Fig. 5. Decision tree, through which the detections are passed to check if they are real. The numbers under the green circles correspond to the number of sources fulfilling the related criteria. See text for a detailed explanation of each step.

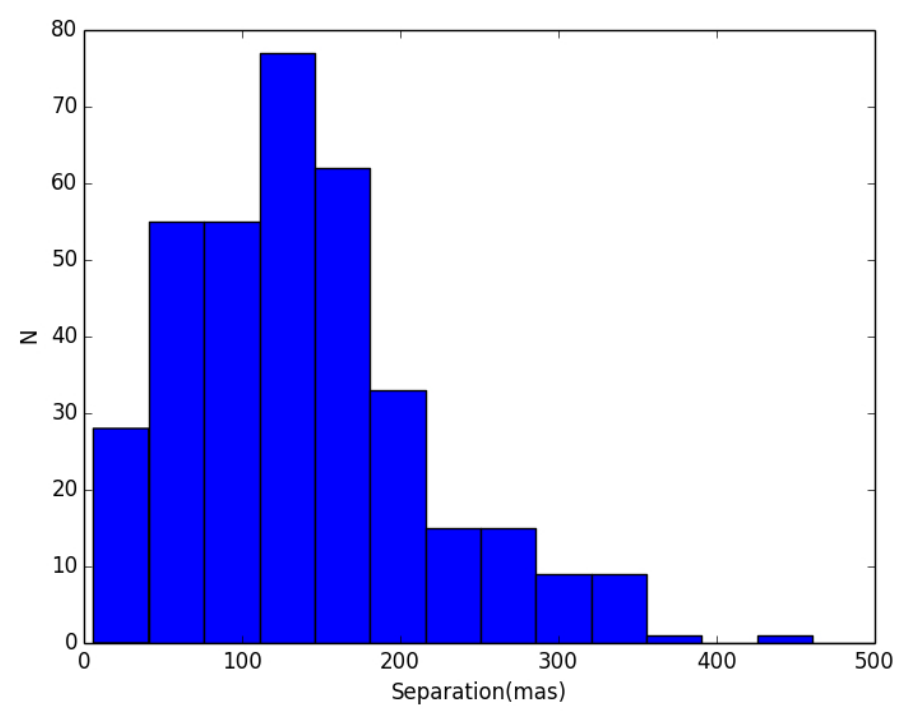

Fig. 6. Number of VLBA detections with $\mathrm{S} / \mathrm{N}$ larger than 7 versus the separation between the VLA source position and the VLBA source position (in milli-arcsec).

- Column (2) - Source name from Schinnerer et al. (2010).

- Column (3) - Integrated VLA flux density of the source (1.4 $\mathrm{GHz}$ ), in $\mu \mathrm{Jy}$, taken from Schinnerer et al. (2010).

- Column (4) - VLBA classification between single- and multi-component source; 0: single-component source; 1 : multi-component source.

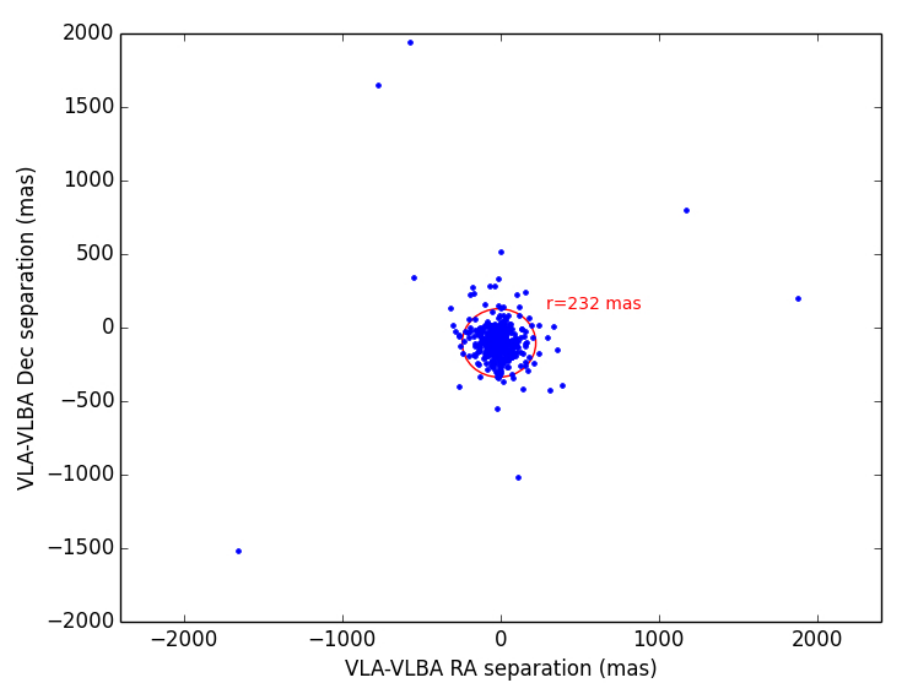

Fig. 7. Relative positions between VLA and VLBA emission. Blue dots show the separation in right ascension and declination between the VLA and the VLBA source position, in mas. The red circle encompasses the $90 \%$ of the detections, with a radius of 232 mas.

- Columns (5) and (6) - Right ascension and declination (J2000) of the source, measured with the VLBA (uniform weighting) in degrees.

- Column (7) - Local noise rms measured in $\mu \mathrm{Jy}^{\text {beam }}{ }^{-1}$ with the VLBA (uniform weighting).

- Columns (8) and (9) - Peak flux density of the source and its error, measured in $\mu \mathrm{Jy}_{\text {beam }^{-1}}$ with the VLBA (uniform weighting).

- Columns (10) and (11) - Integrated flux density of the source and its error (see Sect. 4 for details), measured in $\mu \mathrm{Jy}$ with the VLBA (uniform weighting).

We collected complementary multiwavelenth information (see Table 3 ). We considered counterparts within a radius of $1^{\prime \prime}$. The column entries are the following:

- Column (1) - Source name used in the present project (ID).

- Column (2) - Photometric redshift from Capak et al. (2007), Baldi et al. (2014), Salvato et al. (2011), Kartaltepe et al. (2010a), Brusa et al. (2010) and Lilly et al. (2007).

- Column (3) - Spectroscopic redshift from Gabor et al. (2009), Trump et al. (2009), Civano et al. (2012), Brusa et al. (2010), Kartaltepe et al. (2010b), Lusso et al. (2011), Lackner et al. (2014), Ranalli et al. (2012) and Hao et al. (2014).

- Column (4) - Spitzer/IRAC $3.6 \mu \mathrm{m}$ flux density from Brusa et al. (2010) and Civano et al. (2012), in $\mu \mathrm{Jy}$.

- Column (5) - Spitzer/MIPS $24 \mu \mathrm{m}$ flux density from Brusa et al. (2010), Kartaltepe et al. (2010a) and the Spitzer Enhanced Imaging Products (SEIP) source list from the NASA/IPAC Infrared Science Archive ${ }^{10}$, in $\mu \mathrm{Jy}$.

- Columns (6) and (7) - Soft (0.5-2 keV) and hard (2$10 \mathrm{keV}$ ) band fluxes from Civano et al. (2016), Brusa et al. (2010), Cappelluti et al. (2009) and Hasinger et al. (2007), in $10^{-7} \mathrm{~W} / \mathrm{cm}^{2}$

- Column (8) - Morphological classification from Tasca et al. (2009), Baldi et al. (2014), Salvato et al. (2011), Trump et al. (2009), Brusa et al. (2010), Gabor et al. (2009) and Lusso et al. (2011); 1: early type; 2: spiral; 3: irregular;

${ }^{10}$ http://irsa.ipac.caltech.edu/Missions/spitzer.html 
Table 2. COSMOS VLBA detections catalogue (1.4 GHz).

\begin{tabular}{|c|c|c|c|c|c|c|c|c|c|c|}
\hline (1) & COSMOSVLADP & $\begin{array}{l}S_{i, \mathrm{VLA}} \\
{[\mu \mathrm{Jy}]} \\
(3)\end{array}$ & (4) & $\begin{array}{l}\text { RA } \\
\text { [deg] } \\
(5)\end{array}$ & $\begin{array}{l}\text { Dec } \\
\text { [deg] } \\
(6)\end{array}$ & $\begin{array}{l}\mathrm{rms} \\
{[\mu \mathrm{Jy} /} \\
\text { beam }] \\
(7)\end{array}$ & $\begin{array}{l}S_{\mathrm{p}, \mathrm{VLBA}} \\
{[\mu \mathrm{Jy} /} \\
\text { beam }] \\
(8)\end{array}$ & $\begin{array}{l}\Delta S_{\mathrm{p}, \mathrm{VLBA}} \\
{[\mu \mathrm{Jy} /} \\
\text { beam }] \\
(9)\end{array}$ & $\begin{array}{l}S_{i, \mathrm{VLBA}} \\
{[\mu \mathrm{Jy}]} \\
(10)\end{array}$ & $\begin{array}{l}\Delta S_{i, \mathrm{VLBA}} \\
{[\mu \mathrm{Jy}]} \\
(11)\end{array}$ \\
\hline C1641 & $\mathrm{J} 100028.29+024103.3$ & 82740 & 0 & 150.11785 & 2.684271 & 19.7 & 750 & 78 & 833 & 86 \\
\hline $\mathrm{C} 1670$ & $\mathrm{~J} 100029.62+024018.2$ & 326 & 0 & 150.123454 & 2.671707 & 16.5 & 48 & 17 & 73 & 18 \\
\hline C1679 & $\mathrm{J} 100030.13+013918.2$ & 417 & 0 & 150.125598 & 1.655051 & 18.1 & 116 & 22 & 223 & 29 \\
\hline C1689 & $\mathrm{J} 100031.10+014044.1$ & 379 & 0 & 150.129627 & 1.678869 & 18.4 & 173 & 25 & 214 & 28 \\
\hline $\mathrm{C} 1702$ & $\mathrm{~J} 100032.43+022845.7$ & 279 & 0 & 150.135139 & 2.47937 & 13.3 & 94 & 16 & 145 & 20 \\
\hline $\mathrm{C} 1718$ & $\mathrm{~J} 100033.99+022645.8$ & 142 & 0 & 150.14165 & 2.44605 & 12.4 & 104 & 16 & 119 & 17 \\
\hline $\mathrm{C} 1719$ & $\mathrm{~J} 100033.99+023905.1$ & 290 & 0 & 150.141623 & 2.651423 & 14.2 & 179 & 23 & 183 & 23 \\
\hline C1722 & $\mathrm{J} 100034.37+022121.6$ & 517 & 0 & 150.143226 & 2.356021 & 12.3 & 67 & 14 & 73 & 14 \\
\hline $\mathrm{C} 1725$ & $\mathrm{~J} 100034.83+014247.2$ & 345 & 0 & 150.145154 & 1.713091 & 17.5 & 186 & 26 & 205 & 27 \\
\hline C1739 & $\mathrm{J} 100036.02+023937.9$ & 539 & 0 & 150.150112 & 2.660524 & 15.3 & 290 & 33 & 337 & 37 \\
\hline $\mathrm{C} 1740$ & $\mathrm{~J} 100036.05+022830.6$ & 530 & 0 & 150.150222 & 2.475156 & 13.9 & 85 & 16 & 100 & 17 \\
\hline $\mathrm{C} 1750$ & $\mathrm{~J} 100037.65+022949.0$ & 187 & 0 & 150.156882 & 2.496944 & 12.7 & 152 & 20 & 133 & 18 \\
\hline $\mathrm{C} 1758$ & $\mathrm{~J} 100038.25+022327.8$ & 114 & 0 & 150.159364 & 2.391057 & 12.4 & 56 & 14 & 111 & 17 \\
\hline $\mathrm{C} 1763$ & $\mathrm{~J} 100038.45+024157.6$ & 278 & 0 & 150.160232 & 2.699306 & 18.4 & 106 & 21 & 127 & 22 \\
\hline C1769 & $\mathrm{J} 100039.27+015243.7$ & 115 & 0 & 150.163665 & 1.878767 & 13.9 & 58 & 15 & 61 & 15 \\
\hline $\mathrm{C} 1773$ & $\mathrm{~J} 100039.96+023118.2$ & 132 & 0 & 150.166518 & 2.521681 & 12.6 & 71 & 14 & 72 & 15 \\
\hline $\mathrm{C} 1774$ & $\mathrm{~J} 100040.00+023131.0$ & 55 & 0 & 150.166629 & 2.525287 & 12.4 & 61 & 14 & 55 & 14 \\
\hline $\mathrm{C} 1779$ & $\mathrm{~J} 100040.86+020431.2$ & 131 & 0 & 150.170255 & 2.075272 & 14.4 & 97 & 17 & 77 & 16 \\
\hline $\mathrm{C} 1780$ & $\mathrm{~J} 100040.91+021307.7$ & 213 & 0 & 150.170455 & 2.218779 & 14.1 & 66 & 16 & 75 & 16 \\
\hline $\mathrm{C} 1781$ & $\mathrm{~J} 100041.16+020502.7$ & 203 & 0 & 150.171474 & 2.084064 & 14.0 & 65 & 15 & 92 & 17 \\
\hline $\mathrm{C} 1784$ & $\mathrm{~J} 100041.41+023124.1$ & 716 & 0 & 150.172571 & 2.523339 & 14.1 & 93 & 17 & 127 & 19 \\
\hline $\mathrm{C} 1798$ & $\mathrm{~J} 100042.39+020939.8$ & 251 & 0 & 150.176651 & 2.161034 & 14.6 & 109 & 18 & 98 & 18 \\
\hline $\mathrm{C} 1810$ & $\mathrm{~J} 100043.17+014607.9$ & 88170 & 0 & 150.179973 & 1.768862 & 19.3 & 1544 & 156 & 2525 & 253 \\
\hline C1819 & $\mathrm{J} 100043.53+022524.4$ & 589 & 0 & 150.181401 & 2.423415 & 12.3 & 173 & 21 & 206 & 24 \\
\hline $\mathrm{C} 1824$ & $\mathrm{~J} 100044.55+013942.2$ & 1803 & 0 & 150.185629 & 1.661681 & 20.1 & 898 & 92 & 1150 & 117 \\
\hline C1833 & $\mathrm{J} 100045.25+015459.0$ & 139 & 0 & 150.188504 & 1.916319 & 14.0 & 71 & 16 & 86 & 16 \\
\hline $\mathrm{C} 1847$ & $\mathrm{~J} 100045.80+020119.0$ & 476 & 0 & 150.190843 & 2.021931 & 15.7 & 278 & 32 & 407 & 44 \\
\hline $\mathrm{C} 1860$ & $\mathrm{~J} 100046.91+020726.5$ & 2204 & 1 & 150.195473 & 2.124031 & 14.8 & & & 1383 & 96 \\
\hline C1860a & $\mathrm{J} 100046.91+020726.5$ & 2204 & 0 & 150.195469 & 2.124033 & 19.4 & 581 & 62 & 783 & 81 \\
\hline $\mathrm{C} 1860 \mathrm{~b}$ & $\mathrm{~J} 100046.91+020726.5$ & 2204 & 0 & 150.195476 & 2.12403 & 20.5 & 193 & 28 & 373 & 43 \\
\hline C1860c & $\mathrm{J} 100046.91+020726.5$ & 2204 & 0 & 150.195485 & 2.124025 & 18.9 & 146 & 24 & 227 & 30 \\
\hline $\mathrm{C} 1875$ & $\mathrm{~J} 100047.60+015910.3$ & 21470 & 0 & 150.198312 & 1.986288 & 17.7 & 233 & 29 & 290 & 34 \\
\hline C1884 & $\mathrm{J} 100048.53+013914.0$ & 147 & 0 & 150.202244 & 1.653855 & 19.7 & 144 & 24 & 144 & 22 \\
\hline C1886 & $\mathrm{J} 100048.89+023127.5$ & 234 & 0 & 150.203709 & 2.52428 & 12.3 & 122 & 17 & 140 & 19 \\
\hline C1893 & $\mathrm{J} 100049.58+014923.7$ & 15100 & 0 & 150.20663 & 1.82326 & 16.5 & 669 & 69 & 835 & 85 \\
\hline C1895 & $\mathrm{J} 100049.65+014048.9$ & 243 & 0 & 150.20689 & 1.680226 & 18.7 & 76 & 20 & 95 & 21 \\
\hline C1896 & $\mathrm{J} 100049.78+021654.9$ & 1098 & 0 & 150.20742 & 2.281892 & 14.9 & 512 & 54 & 713 & 73 \\
\hline C1897 & $\mathrm{J} 100049.91+020500.0$ & 311 & 0 & 150.207967 & 2.083336 & 14.8 & 111 & 19 & 236 & 28 \\
\hline C1903 & $\mathrm{J} 100050.45+023356.1$ & 610 & 0 & 150.210223 & 2.565572 & 12.7 & 323 & 35 & 396 & 42 \\
\hline C1911 & $\mathrm{J} 100051.21+014027.3$ & 996 & 0 & 150.21342 & 1.674212 & 18.6 & 149 & 24 & 149 & 22 \\
\hline C1938 & $\mathrm{J} 100054.59+020459.5$ & 121 & 0 & 150.227436 & 2.08317 & 13.4 & 114 & 18 & 114 & 17 \\
\hline C1949 & $\mathrm{J} 100055.36+015955.2$ & 124 & 0 & 150.230691 & 1.998687 & 13.2 & 60 & 15 & 110 & 17 \\
\hline C1959 & $\mathrm{J} 100056.08+014347.3$ & 615 & 0 & 150.233688 & 1.729789 & 17.7 & 86 & 20 & 86 & 19 \\
\hline C1975 & $\mathrm{J} 100057.06+022942.9$ & 123 & 0 & 150.237771 & 2.495213 & 12.9 & 108 & 17 & 108 & 16 \\
\hline C1977 & $\mathrm{J} 100057.11+023451.7$ & 347 & 0 & 150.237968 & 2.581038 & 14.1 & 233 & 27 & 240 & 28 \\
\hline C1978 & $\mathrm{J} 100057.16+013217.8$ & 252 & 0 & 150.238212 & 1.538304 & 42.1 & 197 & 46 & 481 & 64 \\
\hline C1983 & $\mathrm{J} 100057.33+020839.0$ & 193 & 0 & 150.238855 & 2.144165 & 15.3 & 82 & 17 & 105 & 19 \\
\hline C1988 & $\mathrm{J} 100057.45+024217.1$ & 1047 & 0 & 150.239409 & 2.704769 & 17.9 & 426 & 46 & 454 & 49 \\
\hline C1995 & $\mathrm{J} 100057.94+015819.3$ & 319 & 0 & 150.241416 & 1.972019 & 13.5 & 65 & 15 & 101 & 17 \\
\hline $\mathrm{C} 2002$ & $\mathrm{~J} 100058.05+015129.0$ & 13260 & 0 & 150.242241 & 1.859517 & 14.1 & 85 & 17 & 164 & 22 \\
\hline
\end{tabular}

Notes. Column (1): source name used in the present project; Col. (2): source name from Schinnerer et al. (2010); Col. (3): integrated VLA flux density of the source (1.4 GHz), in $\mu \mathrm{Jy}$, taken from Schinnerer et al. (2010); Col. (4): VLBA classification between single- and multi-component source, 0: single-component source, 1: multi-component source; Cols. (5), (6): right ascension and declination (J2000) of the source, measured with the VLBA (uniform weighting) in degrees; Col. (7): local noise rms measured in $\mu \mathrm{Jy}_{\text {beam }}{ }^{-1}$ with the VLBA; Cols. (8), (9): peak flux density of the source and its error, measured in $\mu \mathrm{Jy}$ beam ${ }^{-1}$ with the VLBA (uniform weighting); Cols. (10), (11): integrated flux density of the source and its error (see Sect. 4 for details), measured in $\mu \mathrm{Jy}$ with the VLBA (uniform weighting). Fragment to be used as guidance of the content. The full catalogue is available at the CDS. 
Table 3. VLBA-COSMOS multi-wavelength counterparts.

\begin{tabular}{|c|c|c|c|c|c|c|c|c|c|}
\hline (1) & $\begin{array}{l}\text { zphot } \\
\text { (2) }\end{array}$ & $\begin{array}{l}\text { zspec } \\
\text { (3) }\end{array}$ & $\begin{array}{l}\text { F3.6 } \\
{[\mu \mathrm{Jy}]} \\
(4)\end{array}$ & $\begin{array}{l}\mathrm{F} 24 \\
{[\mu \mathrm{Jy}]} \\
(5)\end{array}$ & $\begin{array}{l}\text { SFlux }(0.5-2 \mathrm{keV}) \\
{\left[10^{-15} 10^{-7} \mathrm{~W} / \mathrm{cm}^{2}\right]} \\
(6)\end{array}$ & $\begin{array}{l}\text { HFlux }(2-10 \mathrm{keV}) \\
{\left[10^{-15} 10^{-7} \mathrm{~W} / \mathrm{cm}^{2}\right]} \\
(7)\end{array}$ & $\begin{array}{l}\text { Mph } \\
\text { (8) }\end{array}$ & $\begin{array}{l}\log M^{*} \\
{[M \operatorname{sun}]} \\
(9)\end{array}$ & $\begin{array}{l}\log \mathrm{MBH} \\
{[M \text { sun }]} \\
(10)\end{array}$ \\
\hline C1641 & 0.32 & 0.3493 & 323.56 & 131.5 & 4 & 6.1 & 1 & 11.46 & \\
\hline C1670 & 0.33 & & & & 0.7 & 3.7 & 1 & & \\
\hline C1679 & 1.35 & & & & & & 2 & & \\
\hline C1689 & 0.94 & 0.84 & & & & & 1 & & \\
\hline C1702 & & & & 140.2 & & & & & \\
\hline C1718 & 0.93 & 0.9 & & & & & 1 & & \\
\hline C1719 & 1.22 & & & & 0.5 & 2.7 & 2 & & \\
\hline C1722 & & & & & & & & & \\
\hline $\mathrm{C} 1725$ & & & & 638.5 & & & & & \\
\hline C1739 & 1.38 & & & & & & 2 & & \\
\hline $\mathrm{C} 1740$ & 0.79 & 0.6879 & 286.5 & 3876.0 & 3.3 & 50 & 2 & 11.31 & 9.48 \\
\hline $\mathrm{C} 1750$ & 0.84 & 0.671 & 30.25 & & 0.6 & 9.3 & 1 & & \\
\hline $\mathrm{C} 1758$ & 0.98 & & & & & & 2 & & \\
\hline $\mathrm{C} 1763$ & 0.3 & 0.72 & & 702.7 & & & 2 & 11.32 & \\
\hline C1769 & 0.64 & & & & & & 1 & & \\
\hline $\mathrm{C} 1773$ & 0.82 & & & & & & 1 & & \\
\hline C1774 & 0.74 & & & & & & 1 & & \\
\hline C1779 & & & & & & & & & \\
\hline $\mathrm{C} 1780$ & 1.05 & 1.156 & 10.8 & 202.2 & 1.6 & 8.1 & 2 & & \\
\hline $\mathrm{C} 1781$ & 0.73 & & & & & & 2 & & \\
\hline $\mathrm{C} 1784$ & 0.76 & & & & & & 1 & & \\
\hline C1798 & 2.12 & & & & & & & & \\
\hline C1810 & 0.34 & 0.346 & 224.72 & 100.0 & 3.5 & 7.5 & 1 & 10.99 & \\
\hline C1819 & 0.84 & 0.7274 & 46.93 & 120.0 & 0.3 & 5.1 & 1 & 10.75 & 9.29 \\
\hline C1824 & 0.17 & & & & & & 1 & & \\
\hline C1833 & 1.03 & & & & & & 2 & & \\
\hline C1847 & 1.05 & & & & & & 3 & & \\
\hline C1860 & 1.73 & 1.158 & & 7322.0 & & & 2 & 11.31 & \\
\hline C1875 & 0.41 & 0.438 & 145.66 & & 0.7 & 4.3 & 1 & & \\
\hline C1884 & 0.17 & & & & & & 1 & & \\
\hline C1886 & 1.28 & & & & & & 2 & & \\
\hline C1893 & 0.53 & 0.53 & & & & & 1 & & \\
\hline C1895 & 1.32 & 0.7134 & 108.44 & 632 & 1.6 & 13.3 & 3 & & \\
\hline C1896 & 0.94 & 0.88 & & & & & 1 & 10.08 & \\
\hline C1897 & 0.45 & 1.2373 & 190.11 & 3052 & 55 & 75 & 1 & & 8.17 \\
\hline C1903 & 1.46 & & & 393 & & & 2 & & \\
\hline C1911 & 0.22 & 0.166 & & 7850 & & & 1 & 11.1 & \\
\hline C1938 & 0.98 & & & & & & 2 & & \\
\hline C1949 & 1.73 & 2.22 & 10.98 & 812 & 0.4 & 2.5 & 1 & & \\
\hline C1959 & & & & 730 & & & & & \\
\hline C1975 & 2.58 & & & & & & 1 & & \\
\hline C1977 & & & & & 1.1 & 3.1 & & & \\
\hline C1978 & 1.36 & & & 180 & & & & & \\
\hline C1983 & 1.73 & & & & & & 2 & & \\
\hline C1988 & 1.27 & & & & & & 2 & & \\
\hline
\end{tabular}

Notes. Column (1): source name used in the present project; Col. (2): photometric redshift; Col. (3): spectroscopic redshift; Col. (4): Spitzer/IRAC $3.6 \mu \mathrm{m}$ flux density, in $\mu \mathrm{Jy}$; Col. (5): Spitzer/MIPS $24 \mu \mathrm{m}$ flux density, in $\mu \mathrm{Jy}$; Cols. (6) and (7): soft (0.5-2 keV) and hard (2-10 keV) band fluxes, in $10^{-7} \mathrm{~W} / \mathrm{cm}^{2}$; Col. (8): morphological classification, 1: early type, 2: spiral, 3: irregular, 4: possible merger, 5: broad emission line object, 6: narrow emission line object, 7: absorption line galaxies, 8: extended source, 9: compact source, 10: normal/star-forming galaxy, 11: red galaxy, 12: FRI, 13: FRII; Col. (9): stellar mass of the galaxy, in $M_{\odot}$; $\mathrm{Col}$. (10): black hole mass, in $M_{\odot}$. For references see details in Sect. 3 . Fragment to be used as guidance of the content. The full catalogue is available at the CDS.

4: possible merger; 5: broad emission line object (type 1 AGN); 6: narrow emission line object (type 2 AGN and starforming galaxies); 7: absorption line galaxies; 8: extended source; 9: compact source; 10: normal/star-forming galaxy; 11: red galaxy; 12: FRI; 13: FRII.
- Column (9) - Stellar mass of the galaxy from Baldi et al. (2014), Kartaltepe et al. (2010b) and Lusso et al. (2011), in $M_{\odot}$.

- Column (10) - Black hole mass from Trump et al. (2011) and Hao et al. (2014), in $M_{\odot}$. 


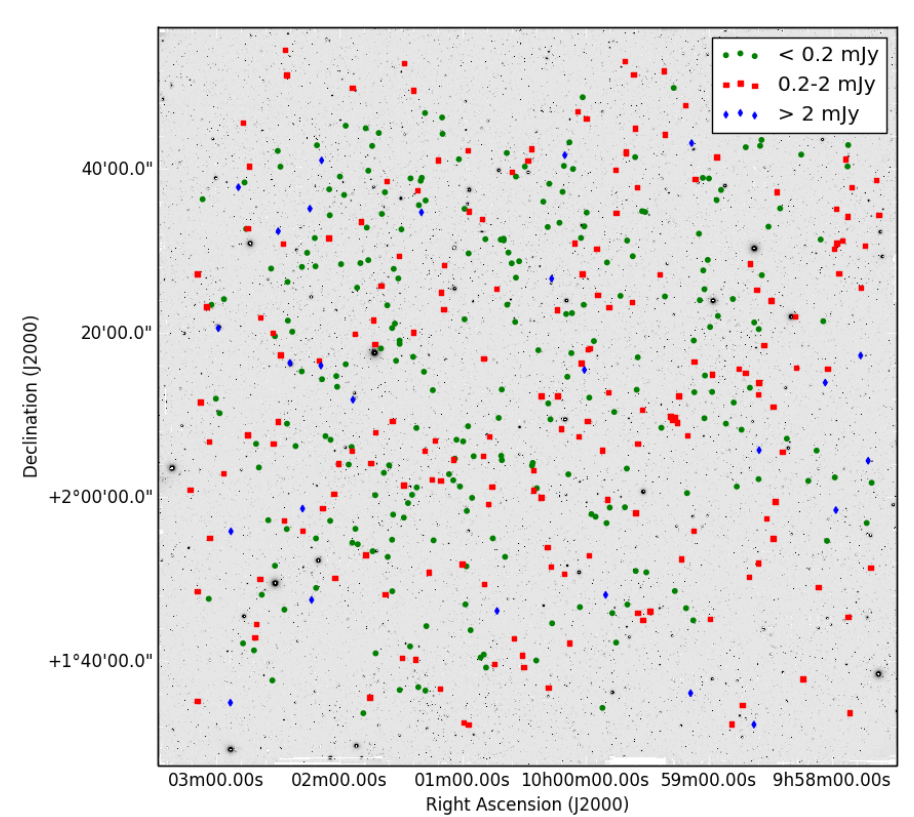

Fig. 8. Distribution of the 468 VLBA detected sources over the COSMOS field. The green circles, red squares and blue diamonds represent VLBA detections with $S_{\text {int }}<0.2 \mathrm{mJy}, 0.2 \mathrm{mJy}<S_{\text {int }}<2 \mathrm{mJy}$ and $S_{\text {int }}>2 \mathrm{mJy}$, respectively. The background greyscale image is a mosaic of the COSMOS Subaru $i$-band data.

Tables 2 and 3 are fragments from the on-line catalogues, available at the CDS, to illustrate them.

False-ID rate. The cross-matching of our radio catalogue with the ancillary data can lead to misidentified sources. Therefore, we have calculated our false identification (false-ID) rate as follows: first, we shifted the positions of all our radio sources by 1 arcmin both in RA and Dec. Second, we cross-matched our new radio catalogue, containing 468 shifted sources, with the catalogue from Capak et al. (2007), since it was the one from where we had the highest number of counterparts (389). Finally, we found counterparts for two radio sources, giving a false-ID rate of around $0.4 \%$.

\section{Results and discussion}

The positions in the COSMOS field of the 468 sources detected by the VLBA are shown in Fig. 8. We can see that the distribution of the sources is roughly homogeneous.

\subsection{Detection fraction}

In the case of VLA multi-components sources detected with the VLBA, only the core of the source was detected. This is reasonable, since the rest of the components are expected to be extended regions, i.e., less likely for a VLBA detection. We searched then for the number of the radio sources from the input catalogue, which would be detectable by the VLBA. We considered a VLA source as detectable by the VLBA when its peak flux density exceeds 5.5 times the local noise level of the VLBA naturally weighted image. Applying this criterion, we found that 2361 out of the 2865 VLA sources were in principle detectable. For this reason, we evaluate the detection fraction based on these 2361 sources.

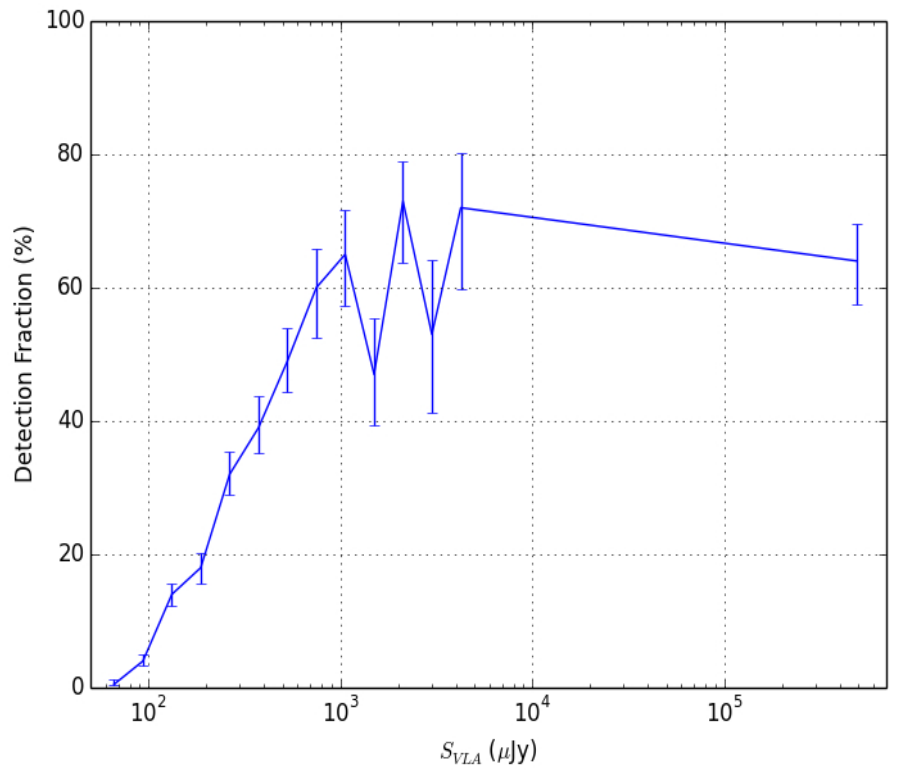

Fig. 9. VLBA detection fraction as a function of $S_{\text {VLA }}$. Only the VLBA detectable sources have been considered to compute this fraction. The VLA flux densities have been separated into bins corresponding to $55 \mu \mathrm{Jy} \times \sqrt{2^{N}}$, where $N=0,1,2, \ldots$ The error bars have been calculated using the Bayesian technique for binomial populations as described by Cameron (2011, $1 \sigma$ confidence interval).

We computed the detection fraction as a function of the VLA flux density $\left(S_{\mathrm{VLA}}\right)$ (see Fig. 9 and Table 4$)$. The uncertainties shown here have been calculated using the Bayesian beta distribution quantile technique as described by Cameron (2011) ( $\sigma \sigma$ confidence interval). It can be seen that, at flux densities $<1 \mathrm{mJy}$, the detection fraction is higher for higher flux densities. At higher flux densities, the detection fraction is independent of flux density. These numbers give us a lower limit on the abundance of AGN in the field, and in particular at faint flux densities, where the achieved sensitivity and the source compactness play a more important role. At high flux densities, a source could be detected even if the VLBI core accounts for a small percentage of the total flux density.

Figure 10 shows the detection fraction as a function of redshift. Photometric redshifts have been used for the redshift bins (see Sect. 3). It can be seen that the detection fraction is roughly constant over the redshift range $0.5<z<3$, showing no evolution. This is in agreement with the findings from Rees et al. (2016). The slight rise of the detection fraction at the highest redshifts probably is due to the small VLBA sample at these redshifts, which make the uncertainties higher.

Table 5 shows the comparison between the number of VLBI detections from various projects (including the present project), grouped into four flux density bins. The Chandra deep field south (CDFS) project achieved a $1 \sigma$ sensitivity of $55 \mu \mathrm{Jy}_{\mathrm{beam}}{ }^{-1}$ and detected $20_{-4}^{+5} \%$ of the sources (Middelberg et al. 2011). The Lockman Hole/XMM project achieved a $1 \sigma$ sensitivity of $24 \mu \mathrm{Jy}_{\text {beam }}{ }^{-1}$ and detected $30 \pm 3 \%$ of the sources (Middelberg et al. 2013). The mJIVE project (Deller \& Middelberg 2014) achieved a $1 \sigma$ sensitivity of $60 \mu \mathrm{Jy}_{\text {beam }^{-1}}$ and detected $20 \pm 0.3 \%$ of the sources. In the present project we achieve a $1 \sigma$ sensitivity of $10 \mu \mathrm{Jy} \mathrm{beam}^{-1}$ and detect $20 \pm 1 \%$ of the sources (468 detections out of 2361 detectable sources). 


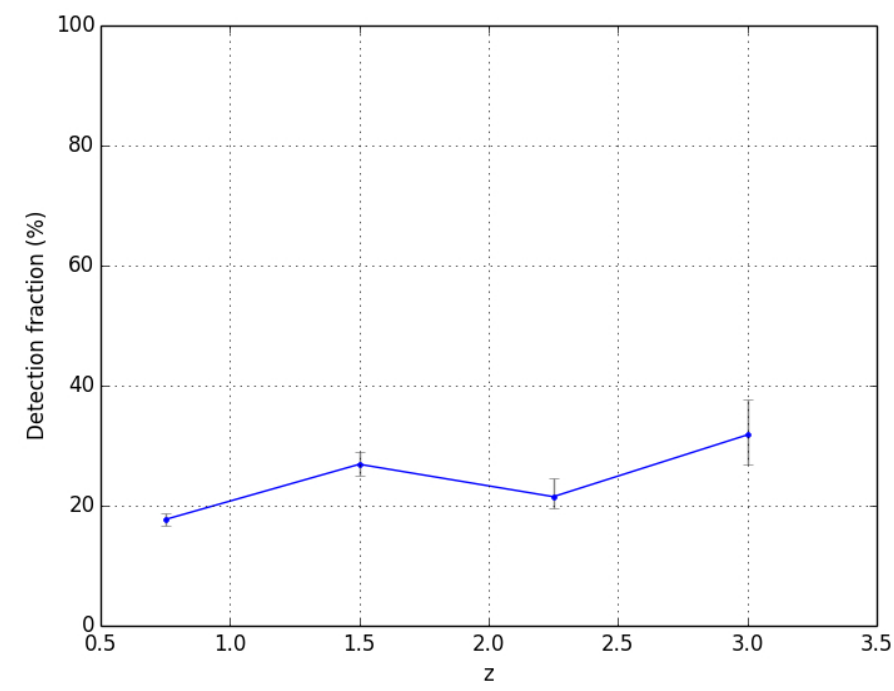

Fig. 10. VLBA detection fraction as a function of redshift. Photometric redshifts have been used (Sect. 3). The error bars have been calculated using the Bayesian technique for binomial populations as described by Cameron (2011, $1 \sigma$ confidence interval).

Table 4. VLBA detection fraction as a function of the VLA flux density.

\begin{tabular}{cccc}
\hline \hline $\begin{array}{c}\text { Flux density bin } \\
(\mu \mathrm{Jy})\end{array}$ & $N_{\mathrm{VLA}}$ & $N_{\text {det }}$ & $\begin{array}{c}\text { Det. fract. } \\
(\%)\end{array}$ \\
\hline $55.0-77.8$ & 382 & 2 & 0.5 \\
$77.8-110.0$ & 578 & 23 & 4 \\
$110.0-155.6$ & 435 & 60 & 14 \\
$155.6-220.0$ & 265 & 47 & 18 \\
$220.0-311.1$ & 203 & 65 & 32 \\
$311.1-440.0$ & 132 & 52 & 39 \\
$440.0-622.3$ & 106 & 52 & 49 \\
$622.3-880.0$ & 52 & 31 & 60 \\
$880.0-1244.5$ & 43 & 28 & 65 \\
$1244.5-1760.0$ & 36 & 17 & 47 \\
$1760.0-2489.0$ & 33 & 24 & 73 \\
$2489.0-3520.0$ & 17 & 9 & 53 \\
$3520.0-4978.0$ & 18 & 13 & 72 \\
$>4978.0$ & 61 & 39 & 64 \\
\hline
\end{tabular}

The targeted sources by the CDFS, Lockman Hole/XMM and mJIVE-20 projects were mainly bright sources, whereas the targeted sources by the VLBA-COSMOS project were mainly faint sources. Since it is assumed that brighter sources are more likely to hold an AGN, the percentage of detected sources with the former three projects are expected to be higher than with our VLBA-COSMOS project. Nevertheless, the sensitivity of our project is much better than those of the other three projects, making the probability to detect a source higher. Therefore, the detection fractions of the four projects are roughly in good agreement with the expectations. One possible explanation of the slightly higher detection fraction of the Lockman Hole/XMM project is the combination of targeting relatively bright sources with the better achieved sensitivity than those achieved by the CDFS and mJIVE-20 projects.

\subsection{VLBA-VLA flux density ratio}

Figure 11 exhibits a diagram showing the VLBA-VLA flux density ratio as a function of the integrated VLA flux density.
Table 5. Number of VLBA detected radio sources by this project, grouped into four flux density bins, compared with the number of detections of different projects.

\begin{tabular}{cccccc}
\hline \hline \multirow{3}{*}{ Project } & & 100 & 0.5 & 1 & \\
& $\mu \mathrm{Jy}$ & $\mu \mathrm{Jy}$ & $\mathrm{mJy}$ & $\mathrm{mJy}$ & \\
\hline COSMOS & 114 & 246 & 57 & 45 & \\
mJIVE & 0 & 8 & 307 & 3679 & $\mathrm{D} 14$ \\
Lockman & 0 & 35 & 12 & 17 & $\mathrm{M} 13$ \\
CDFS & 0 & 1 & 3 & 16 & $\mathrm{M} 11$ \\
\hline
\end{tabular}

References. D14 - Deller \& Middelberg (2014); M13 - Middelberg et al. (2013); M11 - Middelberg et al. (2011).

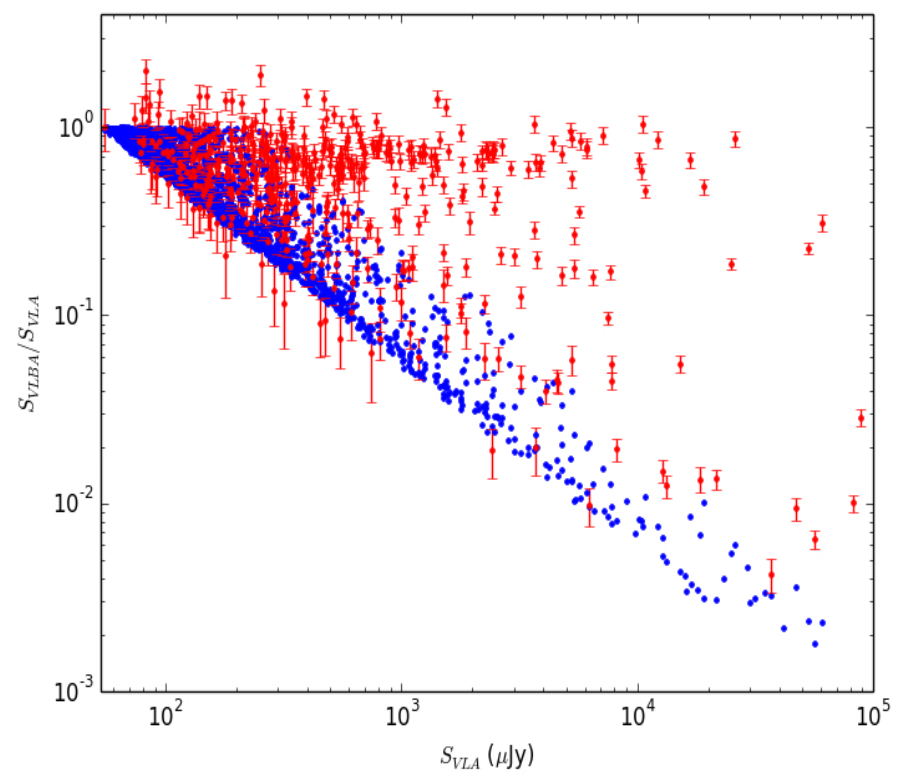

Fig. 11. VLBA-VLA flux density ratio. The blue dots represent upper limits for the VLA sources from the input catalogue. Their VLBA-VLA flux density ratios have been computed as $5.5 \times \mathrm{rms}_{\mathrm{VLBA}} / S_{\mathrm{VLA}}$, where $\mathrm{rms}_{\mathrm{VLBA}}$ is the rms noise of the VLBA naturally-weighted image (since we used the natural weighting for source detection), and $S_{\mathrm{VLA}}$ is the integrated VLA flux density of the source. The red dots with error bars represent the VLBA detected sources.

The median value for the VLBA-VLA flux density ratio of the VLBA detected sources is 0.6. At high VLA flux densities $\left(S_{\mathrm{VLA}}>10 \mathrm{mJy}\right)$, the VLBA-VLA flux density ratio appears to be spread roughly uniformly between 0.01 and 1. At low VLA flux densities ( $S_{\mathrm{VLA}} \lesssim 1 \mathrm{mJy}$ ), it seems that there is an overpopulation of VLBA detected sources with recovered flux densities between $60 \%$ and $80 \%$ of their VLA flux density, suggesting that a large number of low flux density sources detected with the VLBA not only have an AGN but they are also dominated by it. In particular, 237 out of the 344 VLBA detected sources with $S_{\text {VLA }} \lesssim 1$ mJy (69\%) have more than half of their total radio luminosity in a VLBI-scale component, whereas this is true for only six of the 21 VLBA detected sources with $S_{\mathrm{VLA}} \gtrsim 10 \mathrm{mJy}$ (29\%).

To homogenise the samples we should eliminate the bias caused by the fact that at faint flux densities only compact sources can be detected. In order to do so, we considered only sources where the sensitivity of the VLBA observations would allow us to detect a VLBA component (i.e., more than half of 
their total radio luminosity recovered with the VLBA). In this case, the percentage of sources with $S_{\mathrm{VLA}} \lesssim 1 \mathrm{mJy}$ and more than half of their total radio luminosity in a VLBI-scale component shows only a small variation from $69 \%$ to $64 \%$.

These results suggest that low flux density sources have a greater fraction of their radio luminosity in the core and that stronger sources have more extended emission. This is in agreement with the findings from Deller \& Middelberg (2014), which showed that fainter sources were somewhat more likely to be dominated by a very compact component than brighter sources. Mullin et al. (2008) also found a decrease of core prominence with source luminosity and proposed that higher luminosity sources, with faster jets, experience stronger Doppler suppression as an explanation.

Another explanation of why the faint and bright AGN populations differ is that the stronger sources may have large-scale jets and lobes, thus only a smaller fraction of the flux is from the core, whereas the fainter ones tend to be compact. Only $0.9 \%$ of the VLBA detected sources with $S_{\text {VLA }} \lesssim 1 \mathrm{mJy}$ are classified as multi-component in the VLA catalogue of Schinnerer et al. (2010), while this is the case for $50 \%$ of the VLBA detected sources with $S_{\mathrm{VLA}} \gtrsim 10 \mathrm{mJy}$.

The observed difference may also be a consequence of age effects. Many of the faint compact sources presumably are objects similar to the gigahertz peaked-spectrum (GPS) sources, which are thought to be very young and to evolve into the large-scale Fanaroff-Riley class I (FR I) and class II (FR II) radio galaxies (Tinti \& de Zotti 2006). However, the reason behind the high number of observed GPS sources compared to the low number of observed FR I and FR II sources is still not well understood.

It is also worth noting that composite galaxies (with both AGN and star formation playing an important role) are claimed to be a part of the faint radio population (e.g. Strazzullo et al. 2010). Therefore, it is expected that the ratio of compact sources actually drops for the faint sample since the star-formation related radio emission should be resolved out by VLBI. This is the opposite of what we see since we find more compactness in the faint sample of VLBA detected sources, suggesting that the difference between the faint and the bright AGN populations must be even more pronounced because some of it is being masked by the starburst sources.

$4 \%$ of the sources (19/468) have a $S_{\mathrm{VLBA}}$ larger than $S_{\mathrm{VLA}}$ by more than $1 \sigma$ (the length of their error bars). Some of these sources might have larger flux density ratios because of normal Gaussian errors. Three sources out of the $468(0.6 \%)$ have a $S_{\mathrm{VLBA}}$ larger than $S_{\mathrm{VLA}}$ by more than $3 \sigma$. One possible explanation to find VLBA flux densities larger than the VLA flux densities is variability. We note that the VLA observations were performed between 2004 and 2006, while the VLBA observations were performed between 2012 and 2013. Moreover, these sources are towards fainter sources, whose likelihood to be detected increases if they are in a high flux density state.

\subsection{Detected and undetected sources}

We make use of our results and the complementary information in the literature to study the properties of the detected and undetected sources.

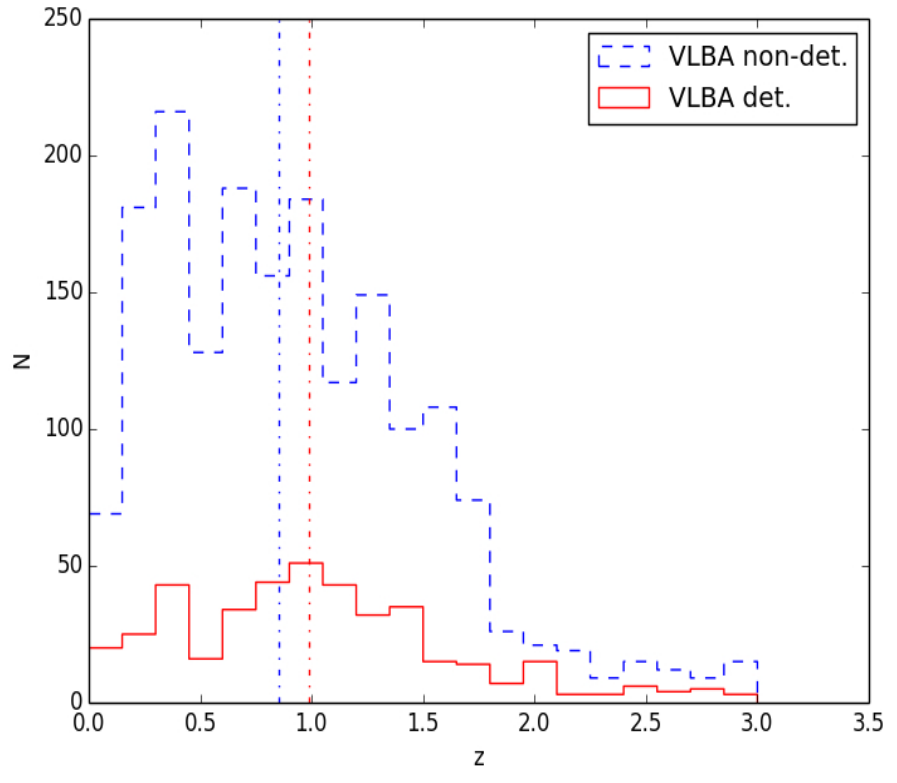

Fig. 12. Number of VLBA detected (red histogram) and undetected sources (blue dashed histogram) as a function of redshift. The vertical dot-dashed lines represent the median photometric redshifts of each sample.

\subsubsection{Redshifts}

We found spectroscopic redshifts of 129 out of 468 VLBA detected sources $(28 \%)$, and 229 out of 2397 VLBA undetected sources $(10 \%)$ (see Sect. 3 for the references). The maximum redshift reached by the detected sources is 3.1 , and by the undetected sources is 2.8 . The median redshift of the detected and undetected sources are 0.71 and 0.59 , respectively. The number of available spectroscopic redshifts is still low. Nevertheless, the available photometric redshifts of the COSMOS field have been demonstrated to have a high accuracy (e.g. Salvato et al. 2011) and therefore are highly reliable.

We have recovered photometric redshifts for 413 out of 468 VLBA detected sources (89\%), and 1799 out of 2397 VLBA undetected sources (75\%) (see Sect. 3 for the references). The maximum redshift achieved by the detected and undetected sources is, in both cases, 3 . The median redshift in the case of detected sources is 0.99 , and in the case of undetected sources is 0.85 . Figure 12 shows the redshift distributions of the VLBA detected and undetected sources.

The median redshift of the VLBA detected sources of $\sim 1$ fortifies the premise that they likely hold an AGN since the brightness temperature of a source to be detected with VLBI observations at that redshift can only be reached by the AGN activity.

We note that even if the total flux density of a source is high, it could not be detected with a high-resolution interferometer if the surface brightness of the source is low. Therefore, one possible explanation of the median redshift of VLBA undetected sources being lower than the median redshift of the VLBA detected sources is that they have low brightness temperatures.

\subsubsection{VLA flux densities}

The median VLA peak flux density of the VLBA detected sources is $320 \mu \mathrm{Jy}$, with a maximum of $24.5 \mathrm{mJy}$ and a minimum of $55 \mu \mathrm{Jy}$, whereas the median VLA peak flux density of the VLBA undetected sources is $100 \mu \mathrm{Jy}$, being the maximum $19.1 \mathrm{mJy}$ and the minimum $27 \mu \mathrm{Jy}$. The low flux density of the 
majority of undetected sources is in agreement with our expectations due to i) a changing underlying source population at lower flux density and ii) the higher need of a very compact core for the fainter sources to be detected.

If we analyse the VLA integrated flux density of the VLBA detected and undetected sources we obtain similar values for the median, $390 \mu \mathrm{Jy}$ and $120 \mu \mathrm{Jy}$, respectively. In this case, the maximum flux density is $88.17 \mathrm{mJy}$ for the detected sources and $175.5 \mathrm{mJy}$ for the undetected sources. The non-detection of the VLA sources with the highest integrated flux densities can be explained as in the previous subsection, since the VLBA could not detect a source of low surface brightness, regardless of its total flux density.

\subsubsection{X-ray fluxes}

We have found X-ray counterparts in the soft band $(0.5-2 \mathrm{keV})$ for 136 VLBA detected sources $(29 \%)$ and 341 undetected sources (14\%), without considering upper limit values. In the case of X-ray fluxes in the hard band $(2-10 \mathrm{keV})$, we found counterparts for 132 VLBA detected sources (28\%) and 337 undetected sources (14\%) (see Sect. 3 for the references).

The median value for the soft band fluxes of the detected and undetected sources is $1.3 \times 10^{-22} \mathrm{~W} / \mathrm{cm}^{2}$ and $1.1 \times 10^{-22} \mathrm{~W} / \mathrm{cm}^{2}$, respectively. The median value for the hard band fluxes of the detected and undetected sources is $6.4 \times 10^{-22} \mathrm{~W} / \mathrm{cm}^{2}$ and $6.7 \times$ $10^{-22} \mathrm{~W} / \mathrm{cm}^{2}$, respectively.

Brandt \& Hasinger (2005) considered AGN those whose soft band flux was larger than $5 \times 10^{-23} \mathrm{~W} / \mathrm{cm}^{2}$. The median value of the fluxes in the soft band found for the VLBA detected sources is above this limit, which strengthens the assumption that they most likely contain an AGN. Nevertheless, the median value of the fluxes in the soft band found for the VLBA undetected sources is also above the limit suggested by Brandt \& Hasinger (2005), suggesting an AGN origin. We find a median value of the VLA integrated flux density for these sources of $120 \mu \mathrm{Jy}$, making them amongst the faintest sources in the sample. At these flux densities sources are only detectable with the VLBA if the majority of their flux density comes from a compact component; this may explain the non-detection of these sources with the VLBA.

On the other hand, we find a relatively low number $(\sim 30 \%)$ of X-ray counterparts for the VLBA detected sources. Since $\mathrm{X}$-ray surveys are generally thought to be very efficient in finding AGN (e.g. Mushotzky 2004), this deserves to be discussed. One possible explanation is that a certain type of AGN is detected by radio surveys but not by $\mathrm{X}$-ray surveys. Comptonthick AGN with column densities of $N_{\mathrm{H}}>1.5 \times 10^{24} \mathrm{~cm}^{-2}$ (e.g. Treister et al. 2009) are so heavily obscured that they remain undetected in X-ray surveys. However, they are not very common. Lanzuisi et al. (2015) conducted a search of Comptonthick AGN in the XMM-COSMOS survey and found only ten. Another possibility is that we are looking at weakly-accreting AGN with lower accretion rates than the X-ray detected sources since it has been argued that hot-mode AGN (radiatively inefficient) do not produce the X-ray characteristics of a typical AGN (e.g. Hardcastle et al. 2006).

Smolčić et al. (2017a) presented the VLA-COSMOS $3 \mathrm{GHz}$ Large Project with which they observed the two square degree COSMOS field with the VLA at $3 \mathrm{GHz}$. Smolčić et al. (2017b) studied the composition of the faint radio population selected from their VLA-COSMOS $3 \mathrm{GHz}$ Large Project and classified the radio sources as star forming galaxies or AGN. They further separate the AGN into moderate-to-high radiative luminosity

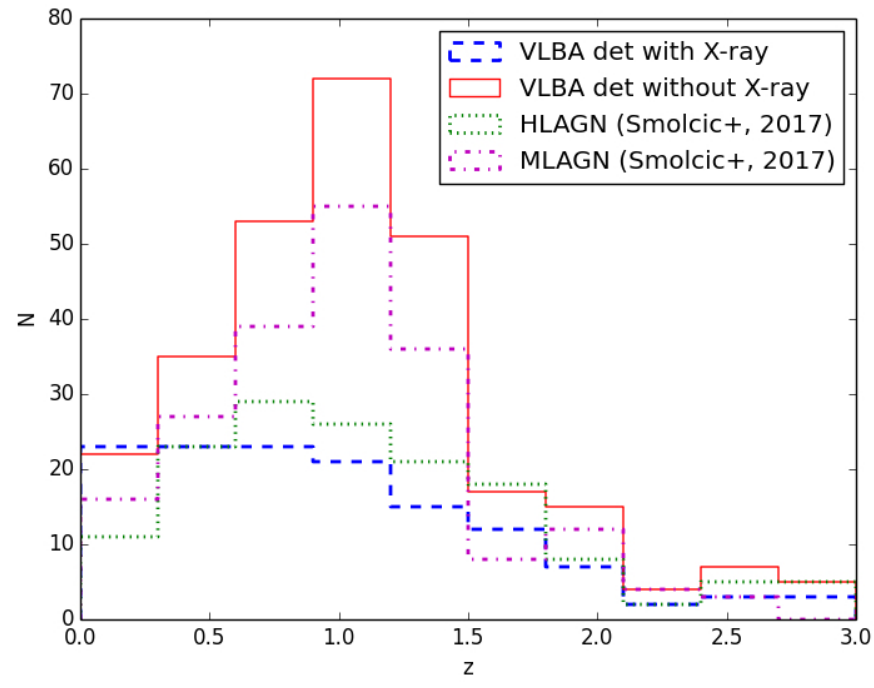

Fig. 13. Redshift distribution of the VLBA detected sources with X-ray counterparts (dashed blue line) and without X-ray counterparts (solid red line). The VLBA detected sources classified either as HLAGN or as MLAGN by Smolčić et al. (2017b) are represented by the dotted green line (HLAGN) and the dash-dotted magenta line (MLAGN).

AGN (HLAGN) and low-to-moderate radiative luminosity AGN (MLAGN) by using their multiwavelength properties. HLAGN were selected by using a combination of X-ray, mid-infrared colour-colour and SED-fitting (see also Delvecchio et al. 2017). The remaining sample was then classified as MLAGN, via excess of radio emission with respect to the star formation of the host galaxy, though completely silent in both X-rays and midinfrared. We cross-matched our catalogue of VLBA detected sources with the sources classified as HLAGN or MLAGN in the catalogue of Smolčić et al. (2017b). Figure 13 shows the redshift distribution of the VLBA detected sources with and without $\mathrm{X}$-ray counterpart and the VLBA detected sources classified either as HLAGN or as MLAGN by Smolčić et al. (2017b). We can see that the MLAGN are mainly responsible for the bump of the VLBA detected sources without X-ray counterpart at $z<1.5$, possibly explaining why no X-ray counterpart is found for them.

These results suggest that while X-ray surveys are highly efficient in selecting AGN with high accretion rates (radiatively efficient), they may underestimate the number of AGN when radiatively inefficient AGN are considered since they may miss the AGN with low accretion rates and therefore with low radiative luminosities.

\subsubsection{Radio-infrared correlation}

The radio-far-infrared correlation is a tight relation between the radio and far-infrared flux densities of galaxies (Condon 1992). A similar correlation has been demonstrated between the $24 \mu \mathrm{m}$ infrared flux density and the $20 \mathrm{~cm}$ radio flux density (Appleton et al. 2004; Boyle et al. 2007). We have used the VLA flux densities to plot the radio-infrared correlation of our sample. We have assembled $24 \mu \mathrm{m}$ flux densities for 154 VLBA detected sources (33\%) and 1145 undetected sources (48\%) (see Sect. 3 for the references). Figure 14 shows the position of the VLBA detected and undetected sources in the radio-infrared correlation.

The radio-infrared correlation has usually been used to identify AGN when there is a large radio excess, since the relation is thought to arise from star-forming activity. Nevertheless, Roy et al. (1998) analysed the radio-far-infrared correlation 


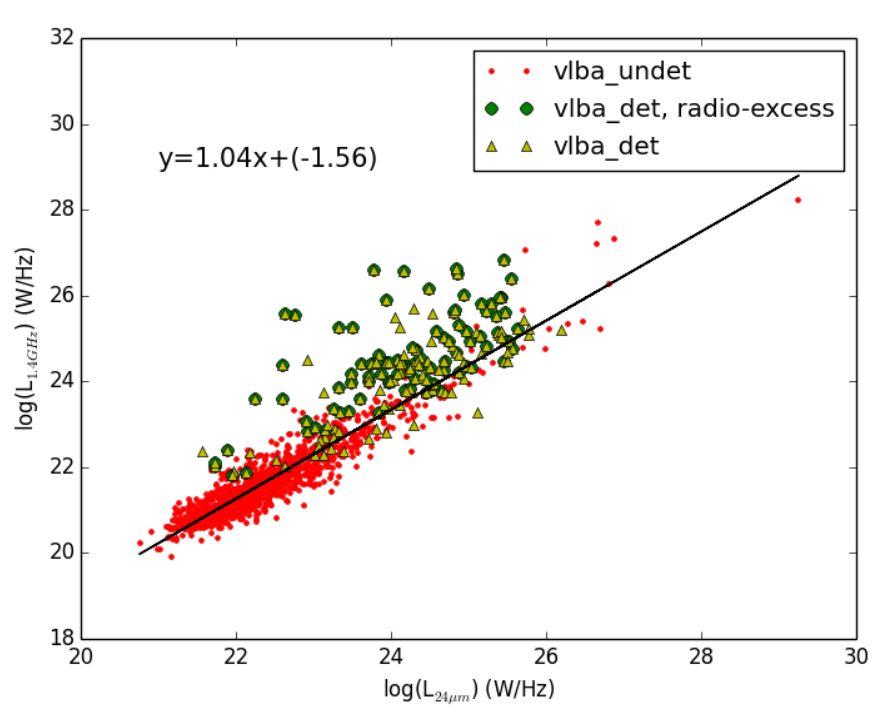

Fig. 14. Radio $(1.4 \mathrm{GHz})$ - infrared $(24 \mu \mathrm{m})$ correlation of the sample, showing the VLBA detected sources classified as radio-excess sources by Smolčić et al. (2017b; see text for details). The VLA flux densities have been used for all the plotted sources. The red dots represent the VLBA undetected sources. The yellow triangles represent the VLBA detected sources. The green circles represent the VLBA detected sources classified as radio-excess sources by Smolčić et al. (2017b) The black line represents the linear regression fitting the VLBA undetected sources.

using a sample of 149 Seyfert galaxies and radio-quiet quasars and found that many Seyferts displayed the same correlation between total radio and far-infrared emission as star-forming galaxies. Additionally, various studies have argued that many low-power AGN appear to obey this relation (Morić et al. 2010; Obrić et al. 2006). Our results are in agreement with them since 53 VLBA detected sources $(34 \%)$ seem to follow the relation within $1 \sigma$. Because the majority of our sample $(\sim 70 \%)$ consists of sub-mJy sources, this method of AGN identification might not be appropriate when the faint radio population is considered.

Smolčić et al. (2017b) used the ratio of the VLA $1.4 \mathrm{GHz}$ radio luminosity and the star formation rate (SFR) in the host galaxy to classify the source as radio-excess source when the ratio showed an excess (see also Delvecchio et al. 2017). The radio emission of the sources classified as radio-excess sources can be attributed to AGN activity. Figure 14 also includes the VLBA detected sources classified as radio-excess sources by Smolčić et al. (2017b). 96 of the 154 VLBA detected sources are also classified as radio-excess sources by Smolčić et al. (2017b). Considering the 53 VLBA detected sources which seem to follow the relation, 20 of them are classified as radio-excess sources. These results suggest that the use of multiple techniques is important to identify all AGN. Amongst these, VLBA observations allow identification of AGN which are not picked up by other methods, and whose exclusion may otherwise lead to a biased view of the AGN population.

\subsubsection{Morphology}

To study the optical morphology of the VLBA detected and undetected sources, we make use of the three classifications described by Tasca et al. (2009): 1) early type; 2) spirals; 3 ) irregulars. We found counterparts for 327 detected sources (70\%) and 1547 undetected sources (65\%). 185 of the detected sources are classified as early type (57\%), 120 as spiral (37\%), and 22 as irregular $(7 \%)$. In the case of the undetected sources, 497 are classified as early type (32\%), 730 as spiral (47\%), and 320 as irregular $(21 \%)$. These results are in agreement with the findings from Middelberg et al. (2013), which suggested that the hosts of VLBI-detected sources are typically early-type or bulgedominated galaxies.

We further separated the sample of the VLBA detected sources with morphological classification into low $(z<1.5)$ and high $(z>1.5)$ redshifts to compare the host optical morphologies. Out of the 283 VLBA detected sources at low redshift, 169 are classified as early type (60\%), 99 as spiral (35\%), and 15 as irregular $(5 \%)$. Out of the 44 VLBA detected sources at high redshift, 16 are classified as early type (36\%), 21 as spiral (48\%), and seven as irregular $(16 \%)$. These results show that, unlike at low redshifts, at $z>1.5$ we found the major classification of host galaxies to be spiral (i.e., star-forming systems). This is in agreement with Rees et al. (2016), who investigated the host galaxy properties of a sample of radio-detected AGN to a redshift of $z=2.25$ and found that the majority of radio-detected AGN at $z>1.5$ are hosted by star-forming galaxies. This finding is interesting because these objects are unusual at low redshifts. One possible explanation is that at higher redshifts we might observe the transition between the starburst produced by a mayor merger and the triggering of the radio-loud AGN (e.g. Seymour et al. 2012), which may lead to the later star formation quenching.

\subsection{VLBA-selected radio AGN and AGN selected by multiwavelength diagnostics}

Delvecchio et al. (2017) analysed a sample of about 7900 radio sources in the COSMOS field observed with the VLA at $3 \mathrm{GHz}$ (Smolčić et al. 2017a) to explore the multiwavelength properties of AGN host-galaxies out to $z \lesssim 6$. They used multiwavelength diagnostics to identify AGN as described in Sect. 4.3.3. To test the robustness of their method, they compared their source classification to other independent methods from the literature. In particular, they cross-matched their $3 \mathrm{GHz}$ VLA catalogue with our $1.4 \mathrm{GHz}$ VLBA catalogue and found that $91 \%$ of the VLBA detected sources were classified as AGN by their method.

The remaining $9 \%$ of the VLBA detected source were misclassified as star-forming galaxies by Delvecchio et al. (2017), probably because they did not show AGN signatures in their multiwavelength properties (X-ray, mid-infrared and SED) and neither did show a significant radio-excess. We note that the threshold above which a radio excess was considered as significant by Delvecchio et al. (2017) was rather conservative (about a factor of 5-6). By looking at those misclassified VLBA sources, they verified that most of them displayed systematically higher radio emission compared to the SFR in the host galaxy, but not significant enough to meet their above criterion (Delvecchio et al., priv. comm.).

\subsection{Two-compact-components VLBA sources}

Figure 15 shows two VLBA detected sources, which exhibit a core with two-components and extended emission not aligned with the two components. The appearance is similar to that found by Rodriguez et al. (2006), the only binary supermassive black hole (SMBH) widely accepted. The three possible scenarios explaining the features of these two VLBA detected sources are: i) one component is the core and the other is a knot in the jet; ii) the two components are the two lobes of a GPS radio source; 
C2662, VLA contours from $36.8 \mu \mathrm{Jy}$, VLBA N_weight S/N 109.2

VLA max 1734.3, VLBA max $1136.0(\mu \mathrm{Jy})$. HST Image
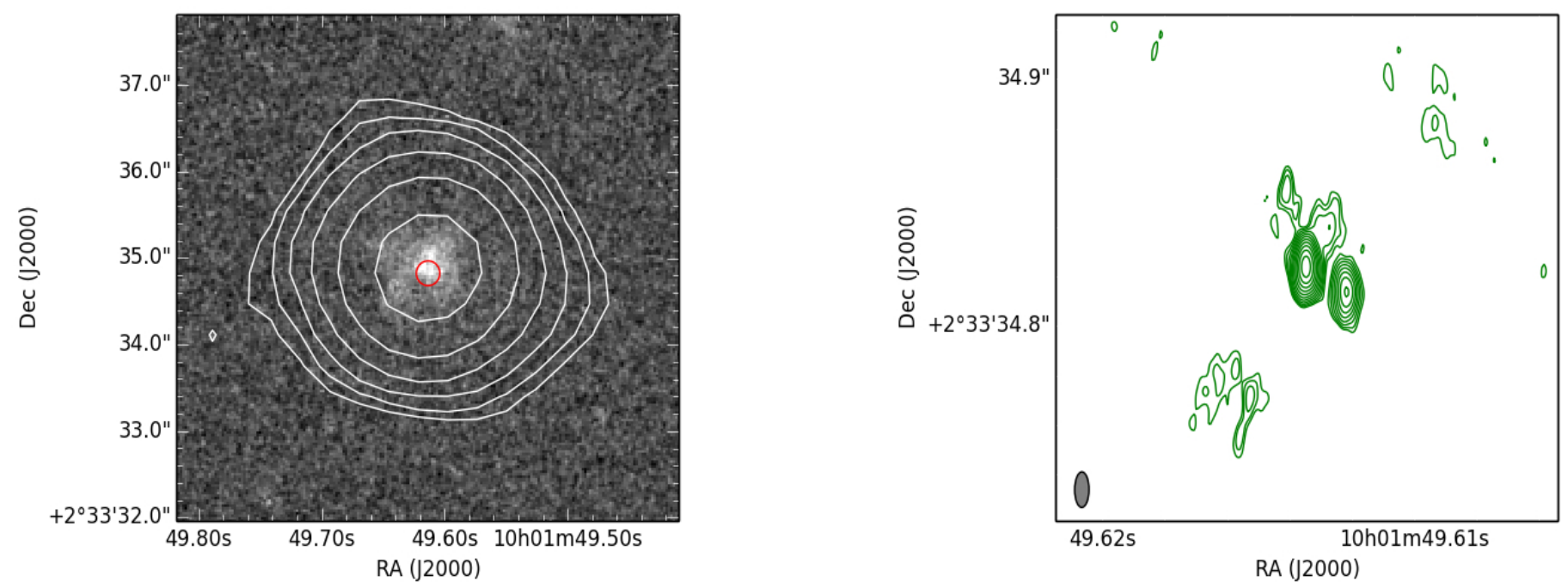

C3026, VLA contours from $53.4 \mu \mathrm{Jy}$, VLBA N_weight S/N 70.6

VLA max 2538.5, VLBA max $903.2(\mu \mathrm{Jy})$. HST Image
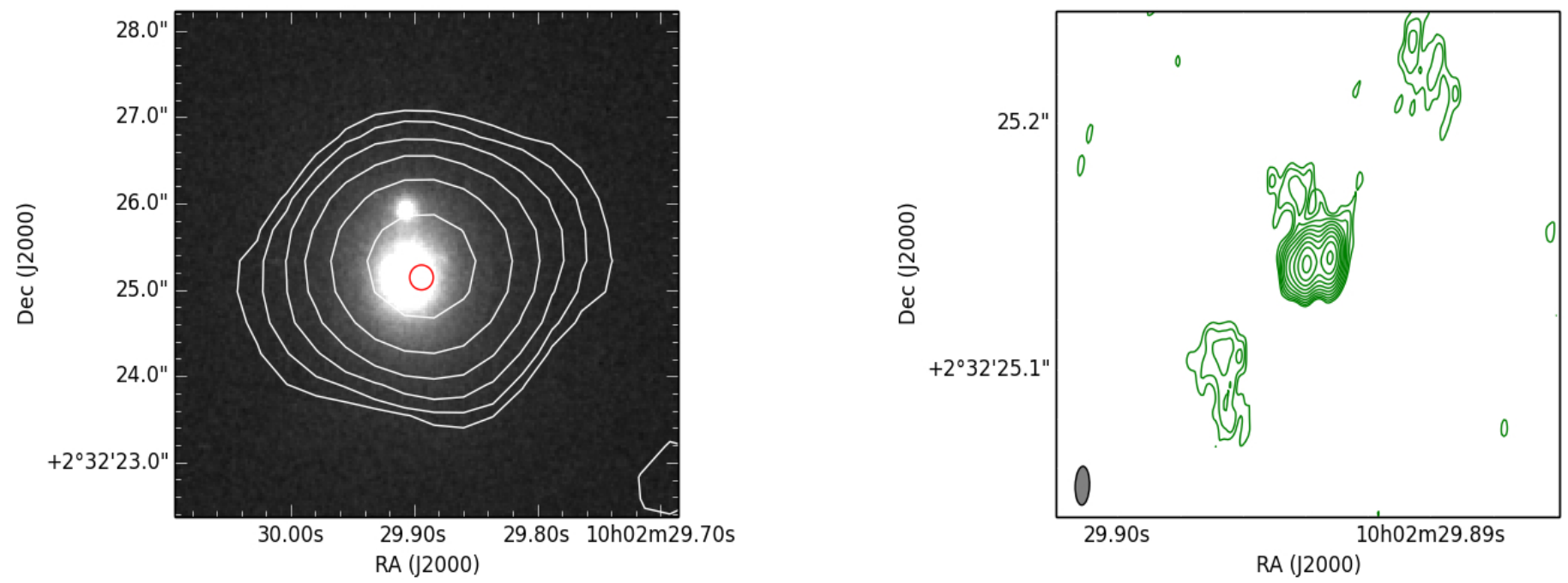

Fig. 15. Two VLBA detected sources, which exhibit a core with two-components. Left panel: background greyscale image is the HST/Subaru image of the VLBA detection counterpart. The white contours represent the VLA contours of the source, starting at four times the rms noise level of the VLA image and increasing by a factor of two. The red circle represents the VLBA peak flux density position. Right panel: green contours represent the VLBA detection contours, starting at three times the rms noise level of the naturally-weighted image and increasing by a factor of $\sqrt{2}$.

or iii) the components are two nuclei involved in a supermassive black hole binary system.

The optical counterpart associated with the radio source C2662 is faint, with a V(AB) magnitude of 24.9. The photometric redshift is 1.4 and the separation between the two components is $176 \mathrm{pc}$. The optical image taken by the Hubble Space Telescope (HST) presents an irregular shape.

The optical counterpart associated with the radio source $\mathrm{C} 3026$ has a $\mathrm{V}(\mathrm{AB})$ magnitude of 21.2. The photometric redshift is 0.43 and the separation between the two components is $63 \mathrm{pc}$. The image taken by the HST shows a normal elliptic shape.

Spectral information is associated with the radio source C2662 from the Magellan Survey (Trump et al. 2007). However, Baldi et al. (2013) found that the object observed by Magellan was not the radio source C2662 (identified by them as "Object
25 "). No spectral information has been found for the candidate C3026.

A proposal to observe these two sources with the VLBA at a lower and a higher frequency to analyse the spectral energy distribution has been accepted in filler time. With these observations we expect to be able to discern between the three aforementioned hypothesis.

\subsection{VLBA-detected radio-quiet quasars}

The origin of the radio emission in radio-quiet quasars (RQQs) has been a matter of discussion for a long time (see Padovani 2016 for a comprehensive review). The two main scenarios ascribe it to either to the star forming activity of the host galaxy (Padovani et al. 2011; Bonzini et al. 2013) or to the black hole 
activity of the AGN (Prandoni et al. 2010). Herrera Ruiz et al. (2016) reported for the first time on a sample of three RQQs where a lower limit of the radio emission coming from the AGN has been measured in the present project. The VLBA-measured radio flux densities are between $50 \%$ and $75 \%$ of the VLA flux densities, demonstrating that the radio emission of at least some RQQs is dominated by the black hole activity of the AGN. Maini et al. (2016) have found similar results in a different sample, making this scenario more relevant.

\section{Conclusions}

VLBI observations of 2865 radio sources were carried out with the VLBA at $1.4 \mathrm{GHz}$, obtaining milli-arcsecond resolution and a $1 \sigma \mathrm{rms}$ noise level of $10 \mu \mathrm{Jy}$ in the central part of the field. The following points list the main outcomes of the project:

1. We have detected 468 sources with the VLBA. To date, this is the largest sample of VLBI detected sources in the submJy regime. We have constructed a catalogue of the detected sources, the main objective of which is to be used as an AGN catalogue for future work in conjunction with complementary multi-wavelength data.

2. On average, the VLBA recovered flux density of the detected sources is $60 \%$ of the VLA flux density. This value represents a lower limit on the fraction of radio emission coming from the AGN. Additionally, we found that low flux density sources have a greater fraction of their radio luminosity in the core, suggesting that the faint radio population is indeed different from the brighter sources.

3. The principal argument to consider the detected sources as AGN is the high resolution provided by VLBI observations, which need sources with very high brightness temperatures to be detected. Moreover, after matching our observations with additional ancillary data, we can be mostly sure that our detections hold an AGN, mainly given their redshifts (we found a median photometric redshift of $\sim 1$ for the VLBA detected sources). Nevertheless, no statement can be made for the undetected sources, since with the use of VLBI observations one can only positively identify AGN, without implying that a non-detection is not an AGN. This follows because the AGN can be temporarily switched off or its emission can be below the detection threshold of the observations.

4. The majority of the host galaxies of the VLBA detected sources are classified morphologically as early type galaxies, i.e., ellipticals and lenticulars, while the predominant classification for the VLBA undetected sources is spiral, in agreement with our expectations. Nevertheless, if we consider only the VLBA detected sources at $z>1.5$, the major classification of the host galaxies is spiral.

5. The wide-field VLBI technique represents a powerful tool to distinguish radio source populations, what is very relevant for future observational projects with, for example, the SKA.

This project provides a valuable tool for the statistical analysis of the faint radio sky. Moreover, $25 \mathrm{~h}$ of observations with the Green Bank Telescope (GBT) in addition to the VLBA have been awarded by the NRAO. We have observed one of the pointings of the COSMOS field with maximum sensitivity, to reach the faintest sources. These data together with the data from the present project will be matter of a future publication and will allow us to study the radio source counts from mJy to the tens of $\mu \mathrm{Jy}$ regime, revealing the $\mathrm{AGN}$ component of the faint radio population.

Acknowledgements. N.H.R. acknowledges support from the Deutsche Forschungsgemeinschaft through project MI 1230/4-1. V.S. and I.D. acknowledge the European Union's Seventh Framework programme under grant agreement 337595 (ERC Starting Grant, "CoSMass"). P.N.B. is grateful for support from STFC via grant ST/M001229/1. We wish to thank the anonymous referee for the helpful comments, which have improved this paper. This research made use of Topcat (Taylor 2005), available at http://www.starlink.ac.uk/topcat/. This research also made use of APLpy, an open-source plotting package for Python hosted at http://aplpy.github.com, and Astropy, a community-developed core Python package for Astronomy (Astropy Collaboration et al. 2013). We wish to thank the staff of the VLBA who greatly supported the experimental observations in this project. The VLBA is operated by the Long Baseline Observatory, a facility of the National Science Foundation operated under cooperative agreement by Associated Universities, Inc.

\section{References}

Appleton, P. N., Fadda, D. T., Marleau, F. R., et al. 2004, ApJS, 154, 147 Astropy Collaboration, Robitaille, T. P., Tollerud, E. J., Greenfield, P., et al. 2013, A\&A, 558, A33

Baldi, R. D., Chiaberge, M., Capetti, A., et al. 2013, ApJ, 762, 30 Baldi, R. D., Capetti, A., Chiaberge, M., \& Celotti, A. 2014, A\&A, 567, A76 Best, P. N., Kaiser, C. R., Heckman, T. M., \& Kauffmann, G. 2006, MNRAS, 368, L67

Best, P. N., Ker, L. M., Simpson, C., et al. 2014, MNRAS, 445, 955

Bonzini, M., Padovani, P., Mainieri, V., et al. 2013, MNRAS, 436, 3759 Bower, R. G., Benson, A. J., Malbon, R., et al. 2006, MNRAS, 370, 645 Boyle, B. J., Cornwell, T. J., Middelberg, E., et al. 2007, MNRAS, 376, 1182 Brandt, W. N., \& Hasinger, G. 2005, ARA\&A, 43, 827

Brusa, M., Civano, F., Comastri, A., et al. 2010, ApJ, 716, 348 Cameron, E. 2011, PASA, 28, 128

Capak, P., Aussel, H., Ajiki, M., et al. 2007, ApJS, 172, 99 Cappelluti, N., Brusa, M., Hasinger, G., et al. 2009, A\&A, 497, 635

Chandra, P., \& Frail, D. A. 2012, ApJ, 746, 156

Chi, S., Barthel, P. D., \& Garrett, M. A. 2013, A\&A, 550, A68

Civano, F., Elvis, M., Brusa, M., et al. 2012, ApJS, 201, 30

Civano, F., Marchesi, S., Comastri, A., et al. 2016, ApJ, 819, 62 Condon, J. J. 1992, ARA\&A, 30, 575

Croton, D. J., Springel, V., White, S. D. M., et al. 2006, MNRAS, 365, 11

Deller, A. T., \& Middelberg, E. 2014, AJ, 147, 14

Deller, A. T., Tingay, S. J., Bailes, M., \& West, C. 2007, PASP, 119, 318

Deller, A. T., Brisken, W. F., Phillips, C. J., et al. 2011, PASP, 123, 275

Deller, A. T., Vigeland, S. J., Kaplan, D. L., et al. 2016, ApJ, 828, 8

Delvecchio, I., Smolčić, V., Zamorani, G., et al. 2017, A\&A, 602, A3

Dubois, Y., Gavazzi, R., Peirani, S., \& Silk, J. 2013, MNRAS, 433, 3297

Fomalont, E. 1981, National Radio Astronomy Observatory Newsletter, 3 , 3

Gabor, J. M., Impey, C. D., Jahnke, K., et al. 2009, ApJ, 691, 705

Garrett, M. A., Porcas, R. W., Pedlar, A., Muxlow, T. W. B., \& Garrington, S. T. 1999, New Astron. Rev., 43, 519

Garrett, M. A., Muxlow, T. W. B., Garrington, S. T., et al. 2001, A\&A, 366, L5 Garrett, M. A., Wrobel, J. M., \& Morganti, R. 2005, ApJ, 619, 105

Greisen, E. W. 2003, Information Handling in Astronomy - Historical Vistas, Astrophys. Space Sci. Lib, 285, 109

Hales, C. A., Murphy, T., Curran, J. R., et al. 2012, MNRAS, 425, 979

Hales, C. A., Norris, R. P., Gaensler, B. M., et al. 2014, MNRAS, 441, 2555

Hao, H., Elvis, M., Civano, F., et al. 2014, MNRAS, 438, 1288

Hardcastle, M. J., Evans, D. A., \& Croston, J. H. 2006, MNRAS, 370, 1893

Hasinger, G., Cappelluti, N., Brunner, H., et al. 2007, ApJS, 172, 29

Heckman, T. M., \& Best, P. N. 2014, ARA\&A, 52, 589

Herrera Ruiz, N., Middelberg, E., Norris, R. P., \& Maini, A. 2016, A\&A, 589, L2

Kartaltepe, J. S., Sanders, D. B., Le Floc'h, E., et al. 2010a, ApJ, 709, 572

Kartaltepe, J. S., Sanders, D. B., Le Floc'h, E., et al. 2010b, ApJ, 721, 98

Kettenis, M., van Langevelde, H. J., Reynolds, C., \& Cotton, B. 2006, in Astronomical Data Analysis Software and Systems XV, eds. C. Gabriel, C. Arviset, D. Ponz, \& S. Enrique, ASP Conf. Ser., 351, 497

Kewley, L. J., Heisler, C. A., Dopita, M. A., et al. 2000, ApJ, 530, 704

Lackner, C. N., Silverman, J. D., Salvato, M., et al. 2014, AJ, 148, 137

Lanzuisi, G., Ranalli, P., Georgantopoulos, I., et al. 2015, A\&A, 573, A137

Lenc, E., Garrett, M. A., Wucknitz, O., Anderson, J. M., \& Tingay, S. J. 2008, ApJ, 673, 78

Lien, A., Chakraborty, N., Fields, B. D., \& Kemball, A. 2011, ApJ, 740, 23 
Lilly, S. J., Le Fèvre, O., Renzini, A., et al. 2007, ApJS, 172, 70

Lusso, E., Comastri, A., Vignali, C., et al. 2011, A\&A, 534, A110

Maini, A., Prandoni, I., Norris, R. P., et al. 2016, A\&A, 589, L3

Middelberg, E. 2006, PASA, 23, 64

Middelberg, E., Deller, A., Morgan, J., et al. 2011, A\&A, 526, A74

Middelberg, E., Deller, A. T., Norris, R. P., et al. 2013, A\&A, 551, A97

Morić, I., Smolčić, V., Kimball, A., et al. 2010, ApJ, 724, 779

Mullin, L. M., Riley, J. M., \& Hardcastle, M. J. 2008, MNRAS, 390, 595

Mushotzky, R. 2004, in Supermassive Black Holes in the Distant Universe, ed.

A. J. Barger, Astrophys. Space Sci. Libr., 308, 53

Norris, R. P. 2009, in The Starburst-AGN Connection, eds. W. Wang, Z. Yang,

Z. Luo, \& Z. Chen, ASP Conf. Ser., 408, 334

Norris, R. P., Lenc, E., Roy, A. L., \& Spoon, H. 2012, MNRAS, 422, 1453

Obrić, M., Ivezić, Ž., Best, P. N., et al. 2006, MNRAS, 370, 1677

Padovani, P. 2016, A\&ARv, 24, 13

Padovani, P., Miller, N., Kellermann, K. I., et al. 2011, ApJ, 740, 20

Planck Collaboration XVI. 2014, A\&A, 571, A16

Prandoni, I., de Ruiter, H. R., Ricci, R., et al. 2010, A\&A, 510, A42

Radcliffe, J. F., Garrett, M. A., Beswick, R. J., et al. 2016, A\&A, 587, A85

Ranalli, P., Comastri, A., Zamorani, G., et al. 2012, A\&A, 542, A16

Rees, G. A., Spitler, L. R., Norris, R. P., et al. 2016, MNRAS, 455, 2731
Rodriguez, C., Taylor, G. B., Zavala, R. T., et al. 2006, ApJ, 646, 49

Roy, A. L., Norris, R. P., Kesteven, M. J., Troup, E. R., \& Reynolds, J. E. 1998, MNRAS, 301, 1019

Salvato, M., Ilbert, O., Hasinger, G., et al. 2011, ApJ, 742, 61

Schawinski, K., Lintott, C. J., Thomas, D., et al. 2009, ApJ, 690, 1672

Schinnerer, E., Sargent, M. T., Bondi, M., et al. 2010, ApJS, 188, 384

Scoville, N., Aussel, H., Brusa, M., et al. 2007, ApJS, 172, 1

Seymour, N., Altieri, B., De Breuck, C., et al. 2012, ApJ, 755, 146

Smolčić, V., Zamorani, G., Schinnerer, E., et al. 2009, ApJ, 696, 24

Smolčić, V., Novak, M., Bondi, M., et al. 2017a, A\&A, 602, A1

Smolčić, V., Delvecchio, I., Zamorani, G., et al. 2017b, A\&A, 602, A2

Strazzullo, V., Pannella, M., Owen, F. N., et al. 2010, ApJ, 714, 1305

Tasca, L. A. M., Kneib, J.-P., Iovino, A., et al. 2009, A\&A, 503, 379

Taylor, M. B. 2005, in Astronomical Data Analysis Software and Systems XIV, eds. P. Shopbell, M. Britton, \& R. Ebert, ASP Conf. Ser., 347, 29

Tinti, S., \& de Zotti, G. 2006, A\&A, 445, 889

Treister, E., Urry, C. M., \& Virani, S. 2009, ApJ, 696, 110

Trump, J. R., Impey, C. D., McCarthy, P. J., et al. 2007, ApJS, 172, 383

Trump, J. R., Impey, C. D., Elvis, M., et al. 2009, ApJ, 696, 1195

Trump, J. R., Impey, C. D., Kelly, B. C., et al. 2011, ApJ, 733, 60

Zinn, P.-C., Middelberg, E., Norris, R. P., \& Dettmar, R.-J. 2013, ApJ, 774, 66 